\title{
Effectiveness of Conservative Treatment According to Severity and Systemic Disease in Carpal Tunnel Syndrome: A Systematic Review
}

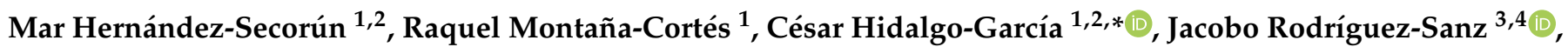 \\ Jaime Corral-de-Toro ${ }^{1,2}$, Sofia Monti-Ballano ${ }^{1,2}$, Sami Hamam-Alcober ${ }^{5}$, José Miguel Tricás-Moreno ${ }^{1,2}$ \\ and María Orosia Lucha-López ${ }^{1,2} \mathbb{D}$ \\ 1 Physiotherapy Department, Faculty of Health Sciences, Universidad de Zaragoza, 50009 Zaragoza, Spain; \\ marhsecorun@unizar.es (M.H.-S.); 722162@unizar.es (R.M.-C.); 682825@unizar.es (J.C.-d.-T.); \\ 681265@unizar.es (S.M.-B.); jmtricas@unizar.es (J.M.T.-M.); orolucha@unizar.es (M.O.L.-L.) \\ 2 Unidad de Investigación en Fisioterapia, University of Zaragoza, 50009 Zaragoza, Spain \\ 3 Department of Basic Sciences, Faculty of Medicine and Health Sciences, Universitat Internacional de \\ Catalunya, 08195 Sant Cugat del Vallès, Spain; jrodriguezs@uic.es \\ 4 ACTIUM Functional Anatomy Group, Universitat Internacional de Catalunya, \\ 08195 Sant Cugat del Vallès, Spain \\ check for \\ updates \\ Citation: Hernández-Secorún, M. \\ Montaña-Cortés, R.; Hidalgo-García, \\ 5 Unit of Reconstructive Surgery of the Locomotor System, Hand-Microsurgery, Department of Orthopaedic \\ Surgery and Traumatology, Hospital Universitario Miguel Servet, 50009 Zaragoza, Spain; \\ jshamam@salud.aragon.es \\ * Correspondence: hidalgo@unizar.es; Tel.: +34-8-7655-4430
} C.; Rodríguez-Sanz, J.; Corral-deToro, J.; Monti-Ballano, S.; HamamAlcober, S.; Tricás-Moreno, J.M. Lucha-López, M.O. Effectiveness of Conservative Treatment According to Severity and Systemic Disease in Carpal Tunnel Syndrome: A Systematic Review. Int. J. Environ. Res. Public Health 2021, 18, 2365. https://doi.org/10.3390/ ijerph18052365

Academic Editor: Paul B. Tchounwou

Received: 2 February 2021

Accepted: 23 February 2021

Published: 28 February 2021

Publisher's Note: MDPI stays neutral with regard to jurisdictional claims in published maps and institutional affiliations.

Copyright: (c) 2021 by the authors. Licensee MDPI, Basel, Switzerland. This article is an open access article distributed under the terms and conditions of the Creative Commons Attribution (CC BY) license (https:// creativecommons.org/licenses/by/ $4.0 /)$
Abstract: (1) Background: Carpal tunnel syndrome (CTS) is the most common peripheral neuropathy in the upper extremity. Conservative treatment has been effective for mild and moderate idiopathic CTS. However, severe CTS and systemic conditions were an exclusion criterion from the studies. The aim of this study is to review the effectiveness of conservative treatment in patients with CTS regardless of the level of severity and the presence or not of systemic diseases in the last ten years. (2) Methods: Randomized controlled clinical trials that compared the effect of conservative treatment on the Boston questionnaire and pain were selected. PubMed, PEDro, Scopus, Cochrane, and Web of Science databases were used. PRISMA statement checklist was performed. (3) Results: 876 studies were recorded, 29 were selected. Pharmacology, Electrotherapy and Manual Therapy had benefits for CTS. Electrotherapy and manual therapy could be effective for severe CTS patients with a systemic condition in the short term, but there was a low percentage of these patients included in the studies. (4) Conclusion: Some pharmacological treatments, manual therapy and electrotherapy have shown benefits for handling CTS, although the most effective combination of techniques is unknown. It would be necessary to include patients with systemic conditions in the selection criteria for future studies.

Keywords: carpal tunnel syndrome; conservative treatment; diabetic neuropathy; electric stimulation therapy; musculoskeletal manipulation; pain

\section{Introduction}

Carpal tunnel syndrome (CTS) is defined as an entrapment of the median nerve in the carpal tunnel in the wrist, generally associated with an increase in carpal tunnel pressure [1] It is the most common peripheral neuropathy in the upper quadrant. The prevalence for CTS ranges from $3.8 \%$ to $4.9 \%$, with women being three times more predisposed than men $[1,2]$. The elevated prevalence of this pathology causes annual healthcare costs to rise, increasing the socioeconomic costs [3].

CTS is characterized by pain and paresthesia in the distributions of the median nerve, including the palmar side of the first finger, the second and third finger and the radial half 
of the fourth finger; it also involves a loss of sensitivity, manual dexterity and functionality. However, the signs and symptoms vary and do not correlate with the level of severity $[1,4]$. In severe cases, weakness of median nerve innervated muscles and spreading symptoms to the forearm, upper arm and sometimes to shoulder could be described. Atrophy of the thenar eminence and weakness of thumb abduction and opposition seem to be the most significant signs $[1,5]$.

Some clinical conditions such as obesity, diabetes and hypothyroidism are linked to a double risk of developing CTS, due to the increased intraneural pressure in the carpal tunnel or vascular deficiencies [6-8]. Different mechanisms have been hypothesized: impaired microvascular circulation for diabetes type 1 and 2 [9]; mucopolysaccharide complex depot for thyroid diseases [10]; hormonal alteration and oedema for menopause and pregnancy [5]; alteration of the fluid balance in the body for obesity [11]. However, more investigation should be done to prove those mechanisms.

In clinical practice guidelines, conservative treatment is recommended for mild and moderate cases, while a surgical approach is recommended for patients with severe CTS [4]. When dealing with patients having severe CTS without diabetic neuropathy, based on thenar muscle atrophy and electrophysiological findings, surgical release is recommended [4]. Even if the results of surgical intervention (carpal tunnel release) are predictable to some extent: about $80-90 \%$ of patients have good to excellent long-term outcomes, remaining $10-20 \%$ have less satisfactory (suboptimal) outcomes, and some of them poor outcome [12]. However, severe symptoms could be present without electrophysiological abnormalities. In a systematic review [13], the effectiveness of the conservative treatment in patients with mild and/or moderate CTS was showed. The use of manual therapy, electrotherapy and pharmacological treatments (the most common techniques nowadays) was described, but no conclusion was given as to which of them was more effective. Other systematic review shows that conservative treatment is effective for short terms but not to longer terms and finally resorting to surgical intervention [14]. These types of treatment were reviewed with the exclusion of patients having severe intensity and/or systemic pathology, conditions that are usually a criterion for exclusion in the sample selection. However, the CTS prevalence in patients with diabetes ranges from $14 \%$ in subjects without diabetic neuropathy, up to $30 \%$ in patients with diabetic neuropathy [15], with a $84 \%$ of risk from suffering CTS during their lifetimes [7]. In addition, even if patients with diabetic neuropathy may improve function and symptoms after CTS surgical release, these subgroup of patients have worst results than CTS patients without this condition [16]. Moreover, they suffer from more complications arising from those procedures. These aspects should be taken into account for treatment election [5,9] for this type of patients and conservative approach could be considered.

We decided to carry out this systematic review because less attention is given to the conservative approach in patients with severe CTS and systemic diseases, there is elevated incidence and prevalence of systemic pathology in CTS, and the fact that CTS involves high individual and socioeconomic costs.

The aim of this study was to review the effectiveness of the conservative treatment in patients having CTS, including severe CTS and patients with systematic diseases in the last 10 years.

\section{Materials and Methods}

\subsection{Protocol and Register}

A systematic review based on the Preferred Reporting Items for Systematic Reviews and Meta-Analyses (PRISMA) statement checklist [17] was designed. The systematic review was registered on the Open Science Framework digital platform: https:/ / osf.io/45q28 / (accessed date 17 February 2021) (DOI 10.17605/OSF.IO/45Q28). 


\subsection{Information Sources and Search}

To create an accurate search strategy, the PICOS (Population, Intervention, Comparison, Outcome and Study design) strategy was taken into account. The Population was people suffering from CTS; the Intervention was conservative treatment such as manual therapy, exercise, electrotherapy or pharmacology; the Comparison was Control, Placebo or other therapies; the Outcome was pain and function; and the Study Design was Randomized Controlled Trial. The following databases were searched: PubMed, Physiotherapy Evidence Database (PEDro), Cochrane Library, SCOPUS and Web of Science. The MESH terms used were "Carpal Tunnel Syndrome", "Therapy"; "Manual Therapy"; "Neural Mobilization" and "Treatment Outcome". Common filters used were language, document type and years. Different search strategies were performed for each database, according to their own filters. The search in PEDro was more concise than the others due to its search method. Boolean operators were used to limit or enlarge the strategy research. Boolean "OR" was used to enlarge the search strategy linking similar terms. Boolean "AND" was used to connect different terms that should be included in search studies in order to limit the search strategy. Table 1 shows the search strategy from each database. The search was performed for dates between 6 September-2 October 2020.

Table 1. Search Strategy.

\begin{tabular}{|c|c|c|}
\hline Database & Search Strategy & Filters \\
\hline PubMed & $\begin{array}{l}\text { "Carpal Tunnel Syndrome" AND (Therapy OR } \\
\text { "Physical Therapy" OR “Manual Therapy" OR } \\
\text { "Neural mobilization") AND Treatment outcome }\end{array}$ & $\begin{array}{l}\text { Text availability: Full text. } \\
\text { Article type: Clinical Trial; } \\
\text { Randomized Controlled Trial. } \\
\text { Publication date: } 10 \text { years. } \\
\text { Language: English, Spanish, French. }\end{array}$ \\
\hline Web of Science & $\begin{array}{l}\text { "Carpal Tunnel Syndrome" AND (Therapy OR } \\
\text { "Physical Therapy" OR “Manual Therapy" OR } \\
\text { "Neural mobilization") AND Treatment outcome }\end{array}$ & $\begin{array}{l}\text { Document type: Article } \\
\text { Timespan: } 2010 \text { to } 2020\end{array}$ \\
\hline SCOPUS & $\begin{array}{l}\text { "Carpal Tunnel Syndrome" AND (Therapy OR } \\
\text { "Physical Therapy" OR “Manual Therapy" OR } \\
\text { "Neural mobilization") AND Treatment outcome } \\
\text { "Carpal Tunnel Syndrome" AND (Therapy OR }\end{array}$ & $\begin{array}{c}\text { Document type: Article } \\
\text { Years: } 2010-2020 \\
\text { Language: English, Spanish, French } \\
\text { Years: } 2010-2020\end{array}$ \\
\hline Cochrane Library & $\begin{array}{l}\text { "Physical Therapy" OR "Manual Therapy" OR } \\
\text { "Neural mobilization") AND Treatment outcome }\end{array}$ & $\begin{array}{c}\text { Trials } \\
\text { Language: English, Spanish, French } \\
\text { Published since: } 2010 .\end{array}$ \\
\hline PEDro & "Carpal Tunnel Syndrome" “Therapy" & $\begin{array}{l}\text { When searching: Match all search terms (AND). } \\
\text { Method: Clinical Trial. }\end{array}$ \\
\hline
\end{tabular}

\subsection{Eligibility Criteria}

The selection of articles included all studies published since 2010 that fulfilled the following criteria: 1 . Randomized controlled clinical trials that compared a conservative treatment group with a control group, a placebo group or a group that received a strictly different treatment; 2 . studies in which the sample included patients diagnosed with idiopathic-type CTS with or without systemic pathology; 3 . studies including pain and functional variables; 4 . studies that evaluated a functional item using the Boston Carpal Tunnel Questionnaire (BCQT), a tool with standardized, patient-based results for the seriousness of the symptoms and the functional status in patients with CTS [18]; 5. studies having a score equal to or more than 6 in the PEDro Scale; 6. studies published in English, French or Spanish.

We excluded the studies in which the sample included the following criteria: 1. pregnant women; 2. patients having suffered traumatisms in the cervical area or upper extremity that might contribute to CTS and its symptoms; 3 . subject with previous surgery and/or surgery during the study period as a CTS treatment. 


\subsection{Study Selection}

Eligible articles and data extraction were conducted independently by two authors (M.H.S. and R.M.C.). Firstly, they evaluated titles and abstracts following the criteria for eligibility, and then complete texts. For any discrepancies or doubts, a third author (C.H.G.) was consulted to resolve the discrepancy.

\subsection{Data Extraction Process}

The following information was extracted for each study: (1) variables analyzed; (5) follow-ups; and (6) results.

\subsection{Risk of Bias in the Individual Studies}

To assess the methodological quality of the clinical trials, the PEDro scale and the Risk of Bias 2 (RoB2) tool in the Cochrane database were used.

The PEDro scale is based on the Delphi list, based on an expert consensus, to help the reader to identify the clinical trials with sufficient internal validity and sufficient statistical information to make their results interpretable. It is formed by 11 criteria to answer using a "Yes" or "No" response, as long as the information is clearly expressed in the study. Each criterion is given 1 point, and the maximum score is 11 points [19].

The RoB2 tool is the second version of the Cochrane tool to assess the risk of bias in clinical trials. The biases are evaluated in 5 domains: (1) randomization process; (2) effect of being assigned to intervention; (3) missing outcome data; (4) measurement of the outcome; (5) reported results. Within each domain, 1 or more questions must be answered. These answers lead to the judgements of "low risk of bias" "some concerns" or "high risk of bias" [20].

\section{Results}

\subsection{Study Selection}

The literature search yielded 876 articles (PubMed: 155, Web of Science: 109; Scopus: 313; PEDro: 62; Cochrane: 237). After we eliminated the duplicated studies, 226 articles were selected for further analysis.

The first analysis focused on the study title and the abstract. We excluded 115 articles because of the study design, sample, presence of surgical intervention, lack of complete article or language different from those of the inclusion criteria.

The last step was analyzing the remaining 111 complete articles to select the ones that fulfilled the inclusion criteria, being finally selected 29 articles for analysis. The selection process is shown in the flow diagram (Figure 1).

\subsection{Study Characteristics \\ 3.2.1. Sample}

The study characteristics are presented in Tables $2-4$. All the articles offered a sample of 1780 upper limbs with CTS. Of these, 28 articles assessed patients with idiopathic CTS, 2 also assessed patients with hypertension and diabetes [21,22] and only 1 article assessed strictly diabetic patients [23]. Additionally, all the studies include mild and moderate severity of CTS, and 8 studies included severe CTS patients [22-29].

To diagnose the presence of CTS and classify the intensity level of the CTS syndrome, most of studies performed an electrophysiological study. However, three of them did not include any neurological study, using only physical examination [25,30,31]. Mostly, the physical examination consisted of the presence of symptoms such as paresthesia and pain in median nerve distribution area, positive Phalen's and Tinel's test. Others test performed was Katz's diagram and the flick sign. The Guideline of the American Academy of Neurology (AAN) was used to classify CTS severity according to neurophysiological findings. 


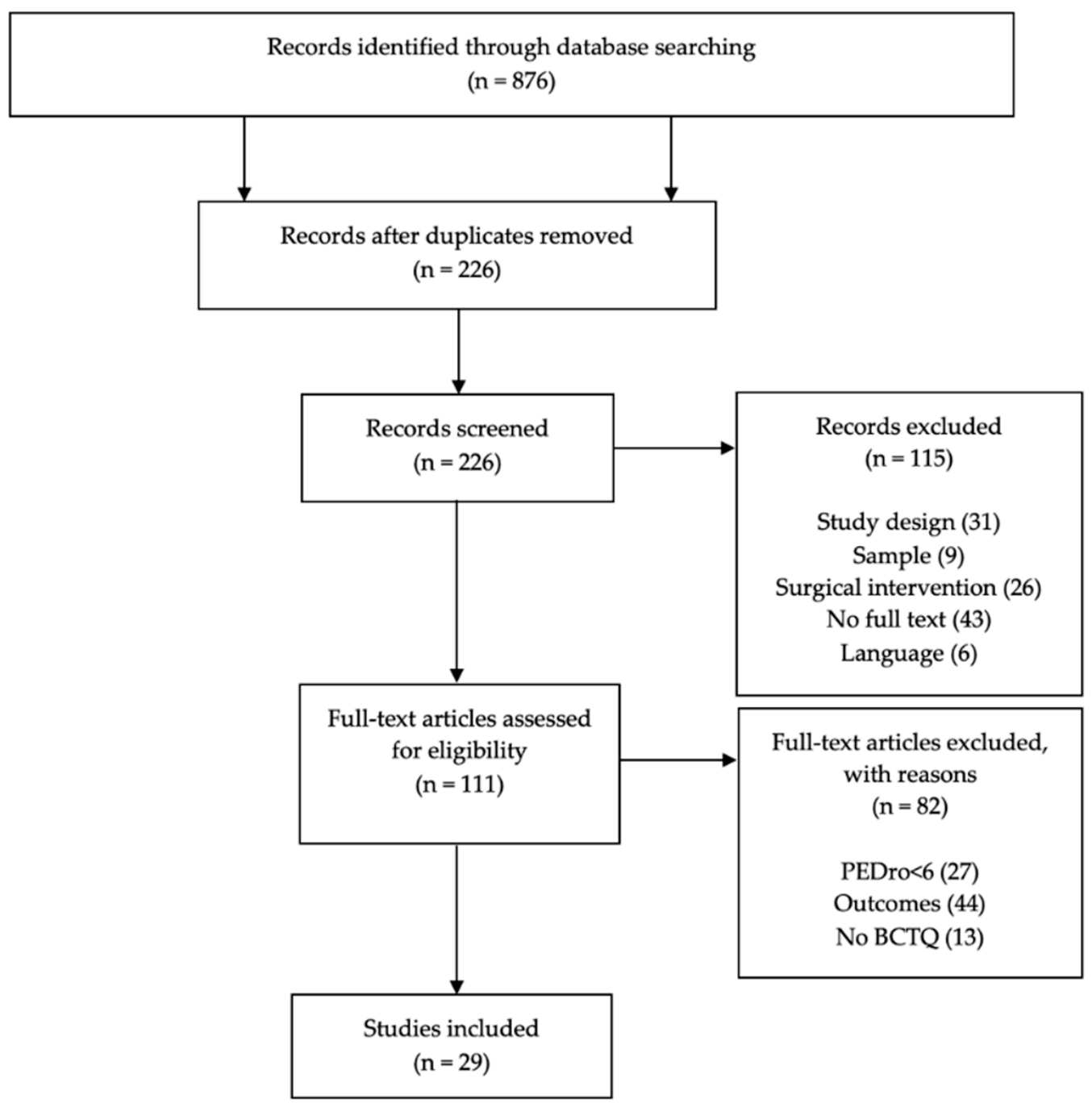

Figure 1. Flow Diagram.

\subsubsection{Intervention}

Six studies observed the effectiveness of drugs [29,32-36]; eight studies evaluated the effectiveness of electrotherapy [21,22,25,37-41] (diathermy, shock waves, radiofrequency, and so on); and eight studies the effect of manual therapy [26-28,42-46]. Three studies compared electrotherapy and a drug intervention $[24,47,48]$ and two studies compared electrotherapy and manual therapy [23,30]. Two articles differed from these categories: one study analyzed the effectiveness of an alternative therapy (lavender ointment) [49] and the other one, a thermal agent (paraffin wax) [50]. Twelve studies had a control group [22,25,27,28,33,41-46]; nine studies a placebo group [21,29,37-40,42,48,49]; and thirteen studies an alternative treatment $[23,24,26,27,30,32,34-37,39,47,48]$. There were six articles having three or more groups $[27,32,37,39,42,48]$.

Applying a splint, applied during night and/or day, was generally the control treatment of choice. The material of the splint was neoprene or a thermoplastic tissue. Some studies provided costumed or prefabricated splints, without describing the kind of material, except for studies that used kinesiotaping. The wrist was placed in neutral position, but this position was only described in two studies (0-5 degrees of wrist extension) $[33,44]$. The educational instruction to explain how to use the splint was considered in a few studies. Only one study combined basic medical care such as ibuprofen with splint treatment [44]. 
Table 2. Characteristics of Studies: Mild and Moderate CTS.

\begin{tabular}{|c|c|c|c|c|c|c|}
\hline \multirow{2}{*}{ Study } & \multicolumn{2}{|r|}{ Sample } & \multirow{2}{*}{ Groups } & \multirow{2}{*}{ Outcomes } & \multirow{2}{*}{ Follow-ups } & \multirow{2}{*}{ Results } \\
\hline & No. $(M / F)$ & CTS Characteristics & & & & \\
\hline $\begin{array}{l}\text { Çatalbas } \\
\text { (2018) }\end{array}$ & $54(8 / 46)$ & $\begin{array}{l}\text { Mild and moderate. } \\
\text { Idiopathic }\end{array}$ & $\begin{array}{l}\text { G1: Continuous ultrasound continuo } \\
\begin{array}{r}(10 \text { sessions } \times 10 \mathrm{~min})+\text { Night splint } \\
(2 \mathrm{wks})\end{array} \\
\text { G2: Pulsed ultrasound (10 sessions } \times \\
10 \mathrm{~min})+ \text { Night splint }(2 \mathrm{wks}) \\
\text { G3: Placebo (Ultrasound- } 10 \text { sessions } \\
\times 10 \mathrm{~min})+ \text { Night splint }(2 \mathrm{wks})\end{array}$ & $\begin{array}{l}B C T Q, V A S \text { (pain and } \\
\text { paraesthesia), Grip } \\
\text { strength, DSL, DML, } \\
\text { SNCV, MNCV, CSA }\end{array}$ & $0,2,6$ weeks & $\begin{array}{l}\text { G1: Improvement in all parameters at } 2 \text { and } \\
\quad 6 \mathrm{wks}[<0167] \\
\text { G2: Improvement in all parameters at } 2 \text { and } \\
\quad 6 \mathrm{wks}[<0167] \\
\text { G3: Improvement in all parameters at } 2 \text { and } \\
6 \text { wks }[<0167] \text { except for grip strength at } 6 \\
\text { wks and DML at } 2 \text { wks. }\end{array}$ \\
\hline $\begin{array}{l}\text { Dinarvand } \\
(2017)\end{array}$ & $37(0 / 37)$ & $\begin{array}{l}\text { Mild and moderate. } \\
\text { Idiopathic }\end{array}$ & $\begin{array}{l}\text { G1: Unciform and scaphoid bone } \\
\text { mobilization }(10 \mathrm{~min}, 3 \mathrm{v} / \mathrm{wk} \times 8 \\
\text { wks }+ \text { Night splint }(8 \mathrm{wks}) \\
\text { G2: Control (Night splint- } 8 \mathrm{wks})\end{array}$ & $\begin{array}{l}\text { VAS (pain), BCTQ, DSL, } \\
\text { DML, SNAP, motor } \\
\text { initiation latency. }\end{array}$ & 0,10 weeks & $\begin{array}{l}\text { G1: Improvement in BCTQ and VAS }[<05] \\
\text { DSL y DML }[<05] . \\
\text { G2: Improvement in BCTQ and VAS }[<05] \\
\text { DSL y DML }[<05] . \\
\text { G1 vs. G2: Improvement in BCQT and VAS } \\
\text { G1 [01]. }\end{array}$ \\
\hline $\begin{array}{c}\text { Eftekharsadat } \\
\text { (2018) }\end{array}$ & $48(7 / 21)$ & $\begin{array}{l}\text { Mild and moderate. } \\
\text { Idiopathic }\end{array}$ & $\begin{array}{l}\text { G1: Lavender ointment }(2 \text { times } / \text { day } \\
\times 40 \text { days })+ \text { Night splint }(40 \mathrm{~d}) \\
\text { G2: Placebo (ointment } 2 \text { times } / \text { day } \times \\
40+\text { night splint }(40 \mathrm{~d})\end{array}$ & $\begin{array}{c}B C T Q, \text { VAS (pain), } \\
\text { Pinch and grip } \\
\text { strength, CMAP, SNAP }\end{array}$ & 0,40 days & $\begin{array}{l}\text { G1: Improvement in BCTQ, VAS, pinch and } \\
\text { grip strength [05]. } \\
\text { G2: Improvement in BCTQ, VAS, pinch and } \\
\text { grip strength [05]. } \\
\text { G1 vs. G2: Improvement in means in G1, ls } \\
\text { pinch strength [05]. }\end{array}$ \\
\hline $\begin{array}{c}\text { Fusakul } \\
\text { (2014) }\end{array}$ & $112(4 / 108)$ & $\begin{array}{l}\text { Mild and moderate. } \\
\text { Idiopathic }\end{array}$ & $\begin{array}{c}\text { G1: Diode laser }(15 \text { sessions } \times 5 \text { wks, } \\
3 \text { times } / \text { wk })+ \text { Splint }(12 \text { wks } \\
\text { morning and night) } \\
\text { G2: Placebo (Laser-15 sessions x } 5 \\
\text { wks, } 3 \text { times } / \text { wk })+ \text { Splint }(12 \text { wks } \\
\text { morning and night) }\end{array}$ & $\begin{array}{l}\text { VAS (pain), BCTQ, } \\
\text { Pinch and grip } \\
\text { strength, Treatment } \\
\text { evaluation, DSL. SNAP, } \\
\text { DML, CMAP }\end{array}$ & 0,5 and 12 weeks & $\begin{array}{c}\text { G1: Improvement in VAS, BCTQ and grip } \\
\text { strength at } 5 \text { and } 12 \text { wks [05]; Pinch } \\
\text { strength and DML at } 12 \text { wks [05]. } \\
\text { G2: Improvement in VAS and BCTQ at } 5 \\
\text { and } 12 \text { wks [05]. Pinch strength and DML } \\
\text { at } 12 \text { wks [05] } \\
\text { G1 vs. G2: Improvement in BCTQ-SSS at } 5 \\
\text { wks [031]. }\end{array}$ \\
\hline
\end{tabular}


Table 2. Cont.

\begin{tabular}{|c|c|c|c|c|c|c|}
\hline \multirow{2}{*}{ Study } & \multicolumn{2}{|c|}{ Sample } & \multirow{2}{*}{ Groups } & \multirow{2}{*}{ Outcomes } & \multirow{2}{*}{ Follow-ups } & \multirow{2}{*}{ Results } \\
\hline & No. (M/F) & CTS Characteristics & & & & \\
\hline $\begin{array}{l}\text { Geler-Külcü } \\
\text { (2016) }\end{array}$ & $60(2 / 58)$ & $\begin{array}{l}\text { Mild and moderate. } \\
\text { Idiopathic }\end{array}$ & $\begin{array}{l}\text { G1: Kinesio-tape ( } 1 \text { time } / 5 \text { days with } \\
\text { 2d rest) + Gliding exercises ( } 4 \mathrm{wks}) \\
\text { G2: Placebo (kinesio-tape- } 1 \text { time } / 5 \\
\text { days with } 2 \text { days of rest) + Gliding } \\
\text { exercises ( } 4 \mathrm{wks}) \\
\text { G3: Night splint (as often as possible) } \\
\quad+\text { Gliding exercises ( } 4 \text { wks) }\end{array}$ & $\begin{array}{l}\text { VAS (pain), DN4 } \\
\text { Questionnaire, BCTQ, } \\
\text { Grip strength. }\end{array}$ & 0,4 weeks & $\begin{array}{c}\text { G1: Improvement in VAS, DN4, BCTQ } \\
\text { [001]. } \\
\text { G2: Improvement in VAS, DN4, BCTQ-SSS } \\
\text { [001]. } \\
\text { G3: Improvement in VAS, DN4, grip } \\
\text { strength, BCTQ-SSS [001]. } \\
\text { G1 vs. G3: Significant difference in favour } \\
\text { of G1 BCTQ [008]. }\end{array}$ \\
\hline $\begin{array}{l}\text { Günay } \\
\text { (2015) }\end{array}$ & $\begin{array}{c}40 \\
(-/-)\end{array}$ & $\begin{array}{l}\text { Mild and moderate. } \\
\text { Idiopathic }\end{array}$ & $\begin{array}{l}\text { G1: Carpal bone mobilization }(10 \\
\text { min/day } \times 3 \text { times } / w k \times 10 \mathrm{~d})+ \\
\text { Splint } \\
\text { G2: Control (Splint) }\end{array}$ & $\begin{array}{l}\text { NPRS (Pain), BCTQ, } \\
\text { Pinch and grip } \\
\text { strength, DSL, DML, } \\
\text { SNAP, CMAP, MNCV, } \\
\text { MCV, EMG. }\end{array}$ & 0,3 months & $\begin{array}{c}\text { G1: Improvement in all clinical variables } \\
\text { [<022]. DSL y SNAP [05]. } \\
\text { G2: Improvement in BCTQ-SSS and NPRS } \\
\text { [01]. } \\
\text { G1 vs. G2: Greatest improvement in G1 } \\
\text { grip strength [04] y BCTQ-FSS [01]. }\end{array}$ \\
\hline $\begin{array}{l}\text { Güner } \\
\text { (2018) }\end{array}$ & $\begin{array}{l}63 \text { hands } \\
(4 / 33)\end{array}$ & $\begin{array}{l}\text { Mild and moderate. } \\
\text { Idiopathic }\end{array}$ & $\begin{array}{l}\text { G1: Low-intensity laser }(5 \text { times } / \text { wk } \\
\quad \times 3 \text { wks } \times 12 \text { min) } \\
\text { G2: Low intensity laser }(5 \text { times } / w k \\
\times 3 \text { wks } \times 12 \text { min })+ \text { Kinesio-tape } \\
\quad(\text { changed every } 2 \mathrm{~d}) \\
\text { G3: Placebo (Laser }-5 \text { times } / \text { wk } \times 3 \\
\quad \text { wks } \times 12 \text { min) }\end{array}$ & $\begin{array}{l}\text { VAS (pain), BCTQ, } \\
\text { Pinch and grip } \\
\text { strength, DML, DSL, } \\
\text { SNCV, MA, SA. }\end{array}$ & $0,3,12$ weeks & $\begin{array}{l}\text { G1: Improvement in VAS, BCTQ, pinch } \\
\text { strength at } 3 \text { and } 12 \text { wks [05]; Grip strength } \\
\text { at } 3 \text { wks [014]; MA and SNCV at } 12 \text { wks } \\
\text { [05]. } \\
\text { G2: Improvement in VAS, BCTQ, pinch and } \\
\text { grip strength at } 3 \text { and } 12 \text { wks [05]; MA, DSL } \\
\text { and SNCV at12 wks [05]. } \\
\text { G3: Improvement in VAS and BCTQ (SSS) } 3 \\
\text { wks [05]; DSL and SNCV at } 12 \text { wks [05]. } \\
\text { G1 vs. G2: Greatest improvement in pinch } \\
\text { and grip strength at } 12 \text { wks [<03] in G2. } \\
\text { G1 vs. G3: Greatest Improvement in VAS } \\
\text { and BCTQ at } 3 \text { and } 12 \text { wks [<01]; Grip } \\
\text { strength at } 3 \text { wks [028] in G1. } \\
\text { G2 vs. G3: All parameters at all times [<03] } \\
\text { in G2. }\end{array}$ \\
\hline
\end{tabular}


Table 2. Cont.

\begin{tabular}{|c|c|c|c|c|c|c|}
\hline \multirow{2}{*}{ Study } & \multicolumn{2}{|c|}{ Sample } & \multirow{2}{*}{ Groups } & \multirow{2}{*}{ Outcomes } & \multirow{2}{*}{ Follow-ups } & \multirow{2}{*}{ Results } \\
\hline & No. (M/F) & CTS Characteristics & & & & \\
\hline $\begin{array}{l}\text { Hadianfard } \\
\quad(2015)\end{array}$ & $\begin{array}{c}50 \\
(3 / 47)\end{array}$ & $\begin{array}{l}\text { Mildand moderate. } \\
\text { Idiopathic }\end{array}$ & $\begin{array}{c}\text { G1: Acupuncture }(2 \text { times } / \text { wk } \times 4 \\
\text { wks })+ \text { Night splint } \\
\text { G2: Control (Night splint+ } \\
\text { Ibuprofen-3 times } / \text { day } \times 10 \mathrm{~d})\end{array}$ & $\begin{array}{l}\text { BCTQ, VAS (pain), } \\
\text { DSL, DML, SNCV. }\end{array}$ & 0,4 weeks & $\begin{array}{c}\text { G1: Improvement in VAS, BCTQ, DSL, } \\
\text { DML, SNCV [<001] } \\
\text { G2: Improvement in VAS, BCTQ, DSL, } \\
\text { DML, SNCV [<001] } \\
\text { G1 vs. G2: Improvement in VAS [<001], } \\
\text { BCTQ [<001], DSL [<001], SNCV [002] }\end{array}$ \\
\hline $\begin{array}{l}\text { Karimzadeh } \\
\qquad(2019)\end{array}$ & $\begin{array}{c}73 \\
(23 / 50)\end{array}$ & $\begin{array}{l}\text { Mild and moderate. } \\
\text { Idiopathic }\end{array}$ & $\begin{array}{l}\text { G1: } 20 \mathrm{mg} \text { Triamcinolone } \\
\text { G2: } 40 \mathrm{mg} \text { Triamcinolone } \\
\text { G3: } 20 \mathrm{mg} \text { Methylprednisolone } \\
\text { G4: } 40 \mathrm{mg} \text { Methylprednisolone }\end{array}$ & $\begin{array}{l}\text { VAS (pain), BCTQ, } \\
\text { Grip strength, CMAP, } \\
\text { SNAP, MNCV. }\end{array}$ & 0,3 months & $\begin{array}{l}\text { G1: Improvement in all variables }[<026] \\
\text { except CMAP. } \\
\text { G2: Improvement in all variables }[<034] \text {. } \\
\text { G3: Improvement in all variables }[<017] \\
\text { except grip strength y CMAP. } \\
\text { G4: Improvements in all variables [<01] } \\
\text { except CMAP. } \\
\text { G1 vs. G2 vs. G3 vs. G4: Significant } \\
\text { difference only in amount in BCTQ-FSS } \\
\text { [005] at } 40 \mathrm{mg} \text {. }\end{array}$ \\
\hline $\begin{array}{l}\text { Kumnerddee } \\
\qquad(2010)\end{array}$ & $\begin{array}{c}60 \\
(6 / 54)\end{array}$ & $\begin{array}{l}\text { Mild and moderate. } \\
\text { Idiopathic }\end{array}$ & $\begin{array}{l}\text { G1: Electro-acupuncture (2 times / wk } \\
\quad \times 5 \mathrm{wks}) \\
\text { G2: Control (Night splint- } 5 \mathrm{wks})\end{array}$ & BCTQ, VAS (pain) & 0,5 weeks & $\begin{array}{c}\text { G1: Improvement in all variables }[<05] . \\
\text { G2: Improvement in BCTQ-SSS [008]. } \\
\text { G1 vs: G2: Significant improvement in VAS } \\
\text { [028] in G1. }\end{array}$ \\
\hline
\end{tabular}


Table 2. Cont

\begin{tabular}{|c|c|c|c|c|c|c|}
\hline \multirow{2}{*}{ Study } & \multicolumn{2}{|c|}{ Sample } & \multirow{2}{*}{ Groups } & \multirow{2}{*}{ Outcomes } & \multirow{2}{*}{ Follow-ups } & \multirow{2}{*}{ Results } \\
\hline & No. $(M / F)$ & CTS Characteristics & & & & \\
\hline $\begin{array}{l}\text { Ordahan } \\
(2017)\end{array}$ & $\begin{array}{c}60 \\
(0 / 60)\end{array}$ & $\begin{array}{l}\text { Mild and moderate. } \\
\text { Idiopathic }\end{array}$ & $\begin{array}{l}\text { G1: Paraffin (5 days/wk } \times 3 \mathrm{wks})+ \\
\text { Splint (always for } 3 \text { wks) }+ \text { Exercises } \\
\quad(2 \text { times/day) } \\
\text { G2: Control (Splint (always for } 3 \mathrm{wks}) \\
\quad+\text { Exercises ( } 2 \text { times/day) })\end{array}$ & $\begin{array}{l}\text { BCTQ, VAS (pain), } \\
\text { DML, DSL, SNAP, } \\
\text { MNCV, CMAP. }\end{array}$ & 0,3 weeks & $\begin{array}{c}\text { G1: Improvement in all variables }[<03] \\
\text { except CMAP. } \\
\text { G2: Improvement in VAS y BCTQ-SSS } \\
\text { [<02]. } \\
\text { G1 vs. G2: Significant improvement } \\
\text { BCTQ-FSS [021]; SNAP [028]; MNCV [041] } \\
\text { in G1. }\end{array}$ \\
\hline $\begin{array}{l}\text { Raeissadat } \\
\text { (2018) }\end{array}$ & $\begin{array}{c}41 \\
(0 / 41)\end{array}$ & $\begin{array}{l}\text { Mild and moderate. } \\
\text { Idiopathic }\end{array}$ & $\begin{array}{c}\text { G1: Leukocyte-poor platelet-rich } \\
\text { plasma (1 injection) + Night splint (8 } \\
\text { wks) } \\
\text { G2: Control (Night splint-8 wks) }\end{array}$ & $\begin{array}{l}\text { BCTQ, VAS (pain), } \\
\text { SNAP, CMAP }\end{array}$ & 0,10 weeks & $\begin{array}{l}\text { G1: Improvement in all variables }[<01] \\
\text { except CMAP. } \\
\text { G2: Improvement in all variables }[<01] \text {. } \\
\text { G1 vs. G2: No significant differences. }\end{array}$ \\
\hline $\begin{array}{l}\text { Senna } \\
(2019)\end{array}$ & $\begin{array}{c}85 \\
(14 / 71)\end{array}$ & $\begin{array}{l}\text { Mild and moderate. } \\
\text { Idiopathic }\end{array}$ & $\begin{array}{l}\text { G1: Platelet-rich plasma (1 dose) } \\
\text { G2: 1ml methylprednisolone acetate } \\
\text { (1 dose })\end{array}$ & $\begin{array}{l}\text { BCTQ, VAS (pain and } \\
\text { paraesthesia), Phalen, } \\
\text { Tinel, DML, CMAP, SL, } \\
\text { SNAP, CSA. }\end{array}$ & 0,1 and 3 months & $\begin{array}{l}\text { G1: Improvement in all variables }[<01] \text { at } \\
\text { all follow-ups. } \\
\text { G2: Improvement in the variables [<01] at } \\
\text { all follow-ups. } \\
\text { G1 vs. G2: No significant differences a } 1 \mathrm{~m} \text {. } \\
\text { Significant improvement to } 3 \mathrm{~m} \text { in } \\
\text { VAS-pain [041]; VAS-paraesthesia [040]; } \\
\text { Phalen [041]; Tinel [039], BCTQ-SSS [007]; } \\
\text { BCTQ-SSS [002]; DML [002]; SL [037]; SC } \\
\text { [049] in G1. }\end{array}$ \\
\hline $\begin{array}{l}\text { Wang } \\
(2017)\end{array}$ & $\begin{array}{c}52 \\
(41 / 11)\end{array}$ & $\begin{array}{l}\text { Mild and moderate. } \\
\text { Idiopathic }\end{array}$ & $\begin{array}{l}\text { G1: Triamcinolone + lidocaine (1 } \\
\text { ultrasound-guided dose) + Splint (as } \\
\text { often as possible for } 12 \mathrm{wks}) \\
\text { G2: Triamcinolone + lidocaine (1 } \\
\text { ultrasound-guided dose) }\end{array}$ & $\begin{array}{l}\text { BCTQ, VAS (pain), } \\
\text { DML, SNCV, CMAP, } \\
\text { SNAP. }\end{array}$ & $0,6,12$ weeks & $\begin{array}{l}\text { G1: Improvement in all variables except } \\
\text { CMAP [<001] } 6 \text { and } 12 \text { wks. } \\
\text { G2: Improvement in all variables except } \\
\text { CMAP [<05] } 6 \text { and } 12 \text { wks. } \\
\text { G1 vs. G2: Significant improvement at } 12 \\
\text { wks in BCTQ-SSS [0032], BCTQ-FSS [.019] } \\
\text { SNCV [015], SNAP [025] in G1. }\end{array}$ \\
\hline
\end{tabular}


Table 2. Cont

\begin{tabular}{|c|c|c|c|c|c|c|}
\hline \multirow{2}{*}{ Study } & \multicolumn{2}{|c|}{ Sample } & \multirow{2}{*}{ Groups } & \multirow{2}{*}{ Outcomes } & \multirow{2}{*}{ Follow-ups } & \multirow{2}{*}{ Results } \\
\hline & No. $(M / F)$ & CTS Characteristics & & & & \\
\hline $\begin{array}{l}\text { Wolny } \\
(2019)\end{array}$ & $\begin{array}{c}103 \\
(11 / 92)\end{array}$ & $\begin{array}{l}\text { Mild and moderate. } \\
\text { Idiopathic }\end{array}$ & $\begin{array}{l}\text { G1: Neurodynamic techniques }(20 \\
\min \times 2 \text { times } / \text { wk } \times 10 \mathrm{wks}) \\
\text { G2: Control }\end{array}$ & $\begin{array}{l}\text { BCTQ, NPRS (pain), } \\
\text { Pinch and grip } \\
\text { strength, SNCV, } \\
\text { MNCV, MT. }\end{array}$ & 0,10 weeks & $\begin{array}{c}\text { G1 vs. G2: Significant improvement SNCV } \\
\text { [<01], MT }[<01] \text {, NPRS }[<01], \text { BCTQ-SSS } \\
{[<01] \text {, BCTQ-FSS }[<01] \text { in G1. }}\end{array}$ \\
\hline $\begin{array}{l}\text { Wolny } \\
(2017)\end{array}$ & $\begin{array}{c}140 \\
(18 / 122)\end{array}$ & $\begin{array}{l}\text { Mild and moderate. } \\
\text { Idiopathic }\end{array}$ & $\begin{array}{c}\text { G1: Neurodynamic techniques }+ \\
\text { Function massage }+ \text { Joint } \\
\text { mobilization }(15 \times 2 \text { times } / \text { wk } \times 10 \\
\text { wks }) . \\
\text { G2: Laser }+ \text { ultrasound }(15 \times 2 \\
\text { times } / \text { wk } \times 10 \mathrm{wks}) .\end{array}$ & $\begin{array}{c}\text { BCTQ, VAS (pain), } \\
\text { SNCV, MNCV, MT, SL }\end{array}$ & 0,10 weeks & $\begin{array}{l}\text { G1: Improvement in all variables }[<01] \\
\text { G2: Improvement in all variables }[<05] \\
\text { except SNCV and MNCV. } \\
\text { G1 vs. G2: Significant improvement in VAS } \\
\text { [<01], BCTQ-SSS [<01], BCTQ-FSS }[<01] \text { in } \\
\text { G1. }\end{array}$ \\
\hline $\begin{array}{c}\text { Wu } \\
(2018)\end{array}$ & $\begin{array}{c}54 \\
(11 / 43)\end{array}$ & $\begin{array}{l}\text { Mild and moderate. } \\
\text { Idiopathic }\end{array}$ & $\begin{array}{l}\text { G1: 5mL Dextrose (1 dose) } \\
\text { G2: 3mL Triamcinolone }\end{array}$ & $\begin{array}{l}\text { VAS (pain), BCTQ, } \\
\text { CSA, SNCV, DML. }\end{array}$ & $0,1,3,4,6$ months & $\begin{array}{l}\text { G1: Improvement in all variables at all } \\
\text { times [<03] except DML at } 1 \text { and } 6 \text { months. } \\
\text { G2: Improvement in VAS, BCTQ-SSS, } \\
\text { BCTQ-FSS } \mathrm{n} 1,3,4 \mathrm{~m}[<01] \text { and VAS } 6 \mathrm{~m} \\
\qquad[<001] \\
\text { G1 vs. G2: Significant improvement VAS at } \\
4 \mathrm{~m}[<01] \text { and } 6 \mathrm{~m}[<001] ; \text { BCTQ-SSS at } 4 \mathrm{~m} \\
{[<01] \text { and } 6 \mathrm{~m}[<001] \text { and BCTQ-FSS at } 4 \mathrm{~m}} \\
\text { and } 6 \mathrm{~m}[<001] \text { in G1. }\end{array}$ \\
\hline $\begin{array}{c}\text { Wu } \\
(2017)\end{array}$ & $\begin{array}{c}60 \\
(10 / 50)\end{array}$ & $\begin{array}{l}\text { Mild and moderate. } \\
\text { Idiopathic. }\end{array}$ & $\begin{array}{l}\text { G1: Perineural injection of } 5 \% \\
\text { dextrose }(1 \text { dose }) \\
\text { G2: Placebo (1 dose of perineural } \\
\text { injection with saline) }\end{array}$ & $\begin{array}{l}\text { VAS (pain), BCTQ, } \\
\text { CSA, SNCV, DML. }\end{array}$ & $0,1,3,6$ months & 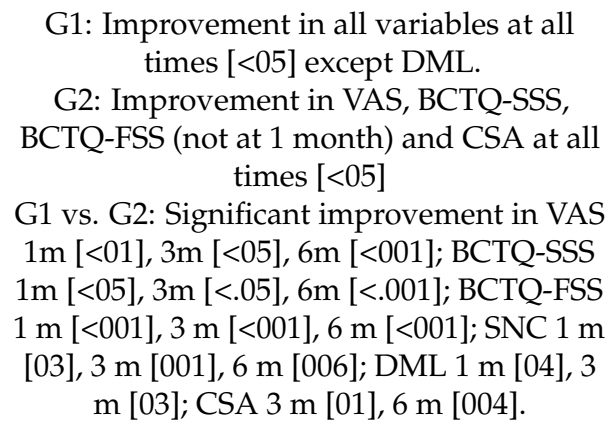 \\
\hline
\end{tabular}


Table 2. Cont.

\begin{tabular}{|c|c|c|c|c|c|c|}
\hline \multirow{2}{*}{ Study } & \multicolumn{2}{|c|}{ Sample } & \multirow{2}{*}{ Groups } & \multirow{2}{*}{ Outcomes } & \multirow{2}{*}{ Follow-ups } & \multirow{2}{*}{ Results } \\
\hline & No. (M/F) & CTS Characteristics & & & & \\
\hline $\begin{array}{c}X u \\
(2020)\end{array}$ & $\begin{array}{c}55 \\
(9 / 46)\end{array}$ & $\begin{array}{l}\text { Mild and moderate. } \\
\text { Idiopathic }\end{array}$ & $\begin{array}{l}\text { G1: Radial extracorporeal shock } \\
\text { waves ( } 1 \text { time/wk } \times 3 \text { wks }) \\
\text { G2: Betamethasone } 1 \mathrm{~mL}(1 \text { dose })\end{array}$ & $\begin{array}{l}\text { VAS (pain), BCTQ, } \\
\text { SNAPa, SNAPdl, } \\
\text { CMAPa, CMAPdl }\end{array}$ & $0,3,9$ and 12 weeks & $\begin{array}{r}\text { G1: Improvement in VAS, BCTQ, SNAPdl } \\
\text { and CMAPdl } 12 \text { wks [<05]; VAS, BCTQ and } \\
\text { SNAPdl } 9 \text { wks [<05]; VAS and BCTQ } 3 \text { wks } \\
\text { [<05]. } \\
\text { G2: Improvement in VAS and BCTQ } 3 \text { wks } \\
\text { [05]; BCTQ } 9 \text { wks [05]. } \\
\text { G1 vs. G2: Significant improvement VAS } 9 \\
\text { wks [<001], } 12 \text { wks [<001]; BCTQ } 9 \text { wks } \\
\text { [<001], } 12 \text { wks [<001]; SNAPdl } 12 \text { wks [004] } \\
\text { in G1. }\end{array}$ \\
\hline $\begin{array}{l}\text { Yildiz } \\
\text { (2011) }\end{array}$ & $\begin{array}{c}44 \\
(8 / 36)\end{array}$ & $\begin{array}{l}\text { Mild and moderate. } \\
\text { Idiopathic }\end{array}$ & $\begin{array}{l}\text { G1: Ultrasound }+ \text { Gel with ketoprofen } \\
(15 \text { min } \times 5 \text { times } / w k \times 2 \text { wks })+ \\
\text { Splint }(8 \text { wks }) \\
\text { G2: Ultrasound }+ \text { Gel with drugs }(15 \\
\text { min } \times 5 \text { times } / w \mathrm{wk} \times 2 \text { wks })+ \text { Splint } \\
(8 \mathrm{wks}) \\
\text { G3: Placebo (ultrasound off }+ \text { gel } \\
\text { without drugs })(15 \text { min } \times 5 \text { times } / \text { wk } \\
\times 2 \text { wks })+ \text { Splint }(8 \text { wks })\end{array}$ & VAS (pain), BCTQ & 0,2 and 8 weeks & $\begin{array}{l}\text { G1: Improvement in all variables } 2 \text { and } 8 \\
\text { wks. } \\
\text { G2: Improvement in all variables } 2 \text { and } 8 \\
\text { wks. } \\
\text { G3: Improvement in all variables } 2 \text { and } 8 \\
\text { wks. } \\
\text { G1 vs. G2: Significant improvement in VAS } \\
8 \text { wks [004] in G1. } \\
\text { G1 vs. G3: Significant improvement in VAS } \\
8 \text { wks [002] in G1. } \\
\text { G2 vs. G3: No significant differences. }\end{array}$ \\
\hline
\end{tabular}

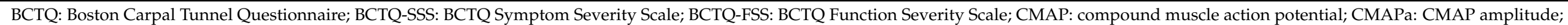

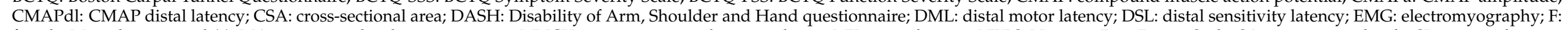

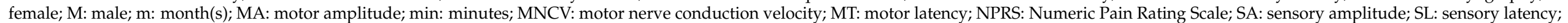

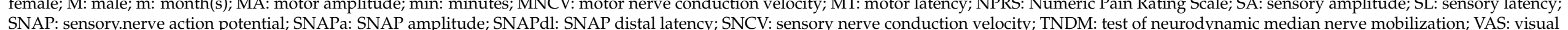
analogue scale; wk(s): week(s). 
Table 3. Characteristics of Studies: Studies Including Severe and Systemic Condition.

\begin{tabular}{|c|c|c|c|c|c|c|}
\hline \multirow{2}{*}{ Study } & \multicolumn{2}{|r|}{ Sample } & \multirow{2}{*}{ Groups } & \multirow{2}{*}{ Outcomes } & \multirow{2}{*}{ Follow-ups } & \multirow{2}{*}{ Results } \\
\hline & No. $(M / F)$ & CTS Characteristics & & & & \\
\hline $\begin{array}{l}\text { Atthakomol } \\
\text { (2018) }\end{array}$ & $\begin{array}{c}25 \\
(6 / 19)\end{array}$ & $\begin{array}{l}\text { Mild, moderate and } \\
\text { severe. Idiopathic }\end{array}$ & $\begin{array}{l}\text { G1: Radial extracorporeal shock waves } \\
\text { ( } 3-7 \text { min }+15 \text { min cold) } \\
\text { G2: Local corticosteroid injections } \\
\text { (triamcinolone, lidocaine) }\end{array}$ & $\begin{array}{l}\text { BCTQ, VAS (pain), } \\
\text { DSL, DML, SNAP, } \\
\text { CMAP }\end{array}$ & $\begin{array}{c}0,1,4,12 \text { and } 24 \\
\text { weeks }\end{array}$ & $\begin{array}{l}\text { G1: Improvement in VAS at } 12 \text { and } 24 \text { wks } \\
\text { [05], BCTQ at } 4 \text { [05], } 12 \text { and } 24 \text { wks [01]; DSL } \\
\text { at } 12 \text { wks [01]. } \\
\text { G2: Improvement in BCTQ at } 1 \text { and } 4 \text { wks } \\
\text { [01]; DSL } 12 \text { wks [01]. } \\
\text { G1 vs. G2: Improvement in BCQT at } 12 \text { a } 24 \\
\text { wks [01] G1; DSL at } 0 \text { and } 12 \text { wks [01] G1. }\end{array}$ \\
\hline $\begin{array}{l}\text { Chen } \\
\text { (2015) }\end{array}$ & $36(1 / 35)$ & $\begin{array}{l}\text { Mild, moderate and } \\
\text { severe. Idiopathic, } \\
\text { hypertension (6) and } \\
\text { diabetics (6) }\end{array}$ & $\begin{array}{l}\text { G1: Pulsed radiofrequency }(32 \mathrm{~min})+ \\
\text { Night splint }(8 \mathrm{~h}) \\
\text { G2: Control (Night splint-8h) }\end{array}$ & $\begin{array}{l}\text { VAS (pain), BCTQ, } \\
\text { CSA, SNCV, Pinch } \\
\text { strength }\end{array}$ & $0,1,4,8,12$ weeks & $\begin{array}{l}\text { G1 vs. G2: All parameters following } \\
\text { intervention }[<05] \text {. Greatest improvement in } \\
\text { VAS and BCTQ at all times, except BCTQ-SSS, } \\
\text { in G1. All parameters improved in both. }\end{array}$ \\
\hline $\begin{array}{l}\text { Chung } \\
\text { (2016) }\end{array}$ & $\begin{array}{c}181 \\
(158 / 23)\end{array}$ & $\begin{array}{l}\text { Mild, moderate and } \\
\text { severe. Idiopathic. }\end{array}$ & $\begin{array}{l}\text { G1: Electro-acupuncture }(13 \text { sessions } \times \\
20 \text { min })+8 \text { h Night splint } \\
\text { G2: Control ( } 8 \text { h Night splint })\end{array}$ & $\begin{array}{l}\text { BCTQ, DASH, NPRS } \\
\text { (pain), Sensitivity, } \\
\text { Manual dexterity, } \\
\text { Pinch strength. }\end{array}$ & $0,1,2,5,17$ weeks & $\begin{array}{l}\text { G1 vs. G2: Improvement in BCTQ-FSS and } \\
\text { DASH [05] at } 1 \text { and } 17 \text { wks. Improvement in } \\
\text { manual dexterity at } 17 \text { wks }[<01] \text {. }\end{array}$ \\
\hline $\begin{array}{l}\text { Horng } \\
(2011)\end{array}$ & $\begin{array}{c}53 \\
(3 / 50)\end{array}$ & $\begin{array}{l}\text { Mild, moderate and } \\
\text { severe. Idiopathic }\end{array}$ & 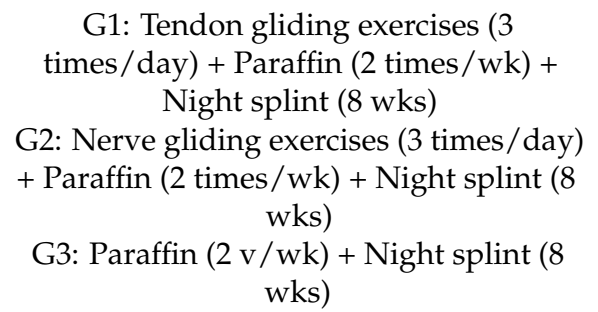 & $\begin{array}{l}\text { BCTQ, VAS (pain), } \\
\text { DASH, WHOQOL (4 } \\
\text { domains). }\end{array}$ & 0,8 weeks & $\begin{array}{c}\text { G1: Improvement in BCTQ, VAS, DASH, } \\
\text { WHOQOL ( } 2 \text { domains) [05]. } \\
\text { G2: Improvement in BCTQ-SSS, VAS, } \\
\text { WHOQOL (1 domain) [05]. } \\
\text { G3: Improvement in BCTQ-SSS, VAS, } \\
\text { WHOQOL (1 domain) [05]. } \\
\text { G1 vs. G2 vs. G3: Significant improvement in } \\
\text { BCTQ-FSS in G1 [04] }\end{array}$ \\
\hline
\end{tabular}


Table 3. Cont.

\begin{tabular}{|c|c|c|c|c|c|c|}
\hline \multirow{2}{*}{ Study } & \multicolumn{2}{|r|}{ Sample } & \multirow{2}{*}{ Groups } & \multirow{2}{*}{ Outcomes } & \multirow{2}{*}{ Follow-ups } & \multirow{2}{*}{ Results } \\
\hline & No. $(M / F)$ & CTS Characteristics & & & & \\
\hline $\begin{array}{l}\text { Talebi } \\
\text { (2018) }\end{array}$ & $\begin{array}{c}30 \\
(-/-)\end{array}$ & $\begin{array}{l}\text { Mild, moderate and } \\
\text { severe. Diabetic }\end{array}$ & $\begin{array}{l}\text { G1: Manual therapy-Nerve }+ \text { interface } \\
\text { mobilization }+ \text { neural }(25 \text { min } \times 3 \\
\text { times } / \text { wk } \times 4 \text { wks }) \\
\text { G2: TENS }(20 \text { min })+\text { Ultrasound }(5 \mathrm{~min}) \\
\quad(3 \text { times } / \text { wk } \times 4 \text { wks })\end{array}$ & $\begin{array}{l}\text { VAS (pain), BCTQ, } \\
\text { TNDM test. }\end{array}$ & 0,4 weeks & $\begin{array}{c}\text { G1: Improvement in all variables }[<009] \\
\text { G2: Improvement in VAS and BCTQ-SSS } \\
{[<001]} \\
\text { G1 vs. G2: Significant improvement in } \\
\text { BCTQ-SSS [006]; BCTQ-FSS [043]; TNDM } \\
{[<001] \text { in G1 }}\end{array}$ \\
\hline $\begin{array}{l}\text { Wu } \\
(2016)\end{array}$ & $\begin{array}{c}40 \\
(5 / 35)\end{array}$ & $\begin{array}{l}\text { Mild, moderate and } \\
\text { severe. Idiopathic, } \\
\text { hypertension (8) and } \\
\text { diabetics (4) }\end{array}$ & $\begin{array}{c}\text { G1: Radial extracorporeal shock waves + } \\
\text { Splint } \\
\text { G2: Placebo (shock waves) + Splint }\end{array}$ & $\begin{array}{l}\text { VAS (pain, } \\
\text { paraesthesia), BCTQ, } \\
\text { CSA, SNCV, Pinch } \\
\text { strength. }\end{array}$ & $1,4,8$ and 12 weeks & $\begin{array}{l}\text { G1: Improvement in all variables at all times } \\
\quad[<01] \text { except SNCV } 1 \mathrm{wk} \text {. } \\
\text { G2: Improvement in all variables at all times } \\
\text { [<05] except BCTQ-FSS } 8 \text { wks and } 12 \text { wks. } \\
\text { G1 vs. G2: Significant improvement in VAS } \\
\text { at } 1 \text { wk [<001], } 4 \text { wks [<001], } 8 \text { wks [003], } 12 \\
\text { wks [006]; BCTQ-SSS at } 1 \mathrm{wk}[017], 4 \text { wks } \\
\text { [005], } 8 \text { wks [008]; BCTQ-FSS at } 1 \mathrm{wk}[001], 4 \\
\text { wks [002], } 8 \text { wks [002], } 12 \text { wks [007]; CSA at } 1 \\
\text { wk [07], } 8 \text { wks [041], } 12 \text { wks [043] in G1. }\end{array}$ \\
\hline
\end{tabular}

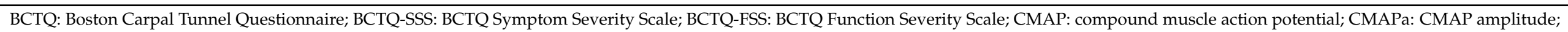

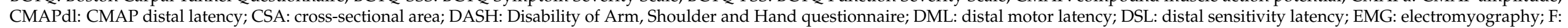

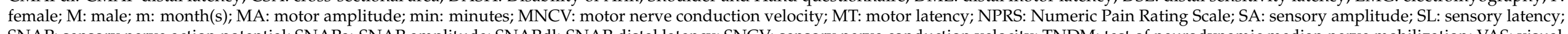

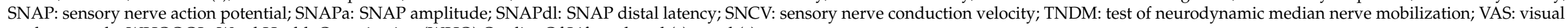
analogue scale; WHOQOL: Word Health Organization (WHO) Quality Of Life scale; wk(s): week(s). 
Table 4. Pain and BCTQ Results of Studies.

\begin{tabular}{|c|c|c|c|c|c|c|c|c|c|}
\hline \multirow{2}{*}{ Group } & \multirow{2}{*}{ Study } & \multirow{2}{*}{ Treatment } & \multirow{2}{*}{\multicolumn{3}{|c|}{ Outcome Pain }} & \multicolumn{4}{|c|}{ Outcome BCTQ } \\
\hline & & & & & & SSS & & & FSS \\
\hline \multirow{3}{*}{ Pharmacological } & $\begin{array}{l}\text { Karimzadeh } \\
\quad \text { (2019) }\end{array}$ & $\begin{array}{l}\text { G1. } 20 \mathrm{mg} \\
\text { Triamcinolone } \\
\text { G2. } 40 \mathrm{mg} \\
\text { Triamcinolone } \\
\text { G3. } 20 \mathrm{mg} \\
\text { Methylpred- } \\
\text { nisolone } \\
\text { G4. } 40 \mathrm{mg} \\
\text { Methylpred- } \\
\text { nisolone }\end{array}$ & $\begin{array}{r}\text { G1. Pre }(5.4 \pm 2 . \\
\text { G2. Pre }(5.4 \pm \\
\text { G3. Pre }(5.6 \pm \\
\text { G4. Pre }(5.3 \pm 2 .\end{array}$ & $\begin{array}{l}\text { VAS } \\
\text { 1) vs. Post }(4 \pm 1.8) p=0.007 \\
\text { 2.4) vs. Post }(3.7 \pm 2.2) p= \\
0.001 \\
\text { 1.8) vs. Post }(4.1 \pm 1.9) p= \\
0.014 \\
\text { ) vs. Post }(3 \pm 2.2) p=0.006\end{array}$ & $\begin{array}{l}\text { Triamcinolone vs. } \\
\text { Methylpred- } \\
\text { nisolone } \\
3.84 \pm 2.1 \text { vs. } 3.53 \\
\pm 2.1(p=0.53) \\
\\
20 \mathrm{mg} \text { vs. } 40 \mathrm{mg} \\
4.05 \pm 1.8 \text { vs. } 3.36 \\
\pm 2.2(p=0.14)\end{array}$ & $\begin{array}{r}\text { G1. Pre }(32.5 \pm 11.8) \text { vs. Pos } \\
=0.002 \\
\text { G2. Pre }(31.7 \pm 8.6) \text { vs. Pos } \\
0.001 \\
\text { G3. Pre }(31.9 \pm 8) \text { vs. Pos } \\
0.001 \\
\text { G4. Pre }(5.3 \pm 2.9) \text { vs. Pos } \\
0.001 \\
\text { Triamcinolone vs. Methy } \\
23.5 \pm 10.5 \text { vs. } 22.6 \pm 7 \\
20 \text { mg vs. } 40 \\
23.88 \pm 9.3 \text { vs. } 22.44 \pm\end{array}$ & $\begin{array}{l}4.9 \pm 10.2) p \\
2.3 \pm 1.1) p= \\
\pm 1.9) p= \\
\pm 2.2) p= \\
\text { dnisolone } \\
p=0.67) \\
(p=0.50)\end{array}$ & $\begin{array}{r}\text { G1. Pre }(32.5 \\
\text { G2. Pre }(31.7 \\
\text { G3. Pre (31. } \\
\text { G4. Pre (5.3 } \\
\text { Triamcinolo } \\
14.0 \pm 4 . \\
16.46 \pm 5.7\end{array}$ & $\begin{array}{l} \pm 11.8) \text { vs. Post }(24.9 \pm 10.2) \\
p=0.002 \\
\pm 8.6) \text { vs. Post }(22.3 \pm 1.1) p \\
=0.001 \\
\pm 8) \text { vs. Post }(.1 \pm 1.9) p= \\
0.001 \\
\pm 2.9) \text { vs. Post }(3 \pm 2.2) p= \\
0.002 \\
\text { ne vs. Methylprednisolone } \\
\text { vs. } 15.3 \pm 5.3(p=0.27) \\
0 \text { mg vs. } 40 \mathrm{mg} \\
\text { vs. } 13.05 \pm 4.4(p=0.005)\end{array}$ \\
\hline & $\begin{array}{c}\text { Raeissadat } \\
\text { (2018) }\end{array}$ & $\begin{array}{c}\text { G1. } \\
\text { Leukocyte-poor } \\
\text { platelet-rich } \\
\text { plasma + Night } \\
\text { splint } \\
\text { G2. Night splint }\end{array}$ & $\begin{array}{r}\text { G1. Pre }(6 \\
\text { G2. Pre (6 } \\
\text { G1 vs. G2-2.90 }=\end{array}$ & $\begin{array}{c}\text { VAS } \\
.82 \pm 1.24) \text { vs. Post }(4.02 \pm 1 . \\
.24 \pm 1.13) \text { vs. Post }(3.52 \pm 2 . \\
2.1 \text { vs. }-2.76 \pm 2.4(p=0.845 \\
0.098 \text { adjusted for age) }\end{array}$ & $\begin{array}{l}\text { 92) } p<0.001 \\
\text { 02) } p<0.001 \\
\text { Power }=0.073 ; p=\end{array}$ & $\begin{array}{r}\text { G1. Pre }(2.43 \pm 0.73) \text { vs. Pos } \\
0.001 \\
\begin{array}{r}\text { G2. Pre }(2.76 \pm 0.40) \text { vs. Pos } \\
<0.001 \\
\text { G1 vs. G2. }-0.05 \pm 0.2 \text { vs. } \\
0.174 ; \text { Power }=0.393 ; p=0 . \\
\text { age }) .\end{array}\end{array}$ & $\begin{array}{l}7 \pm 0.52) p< \\
.90 \pm 0.42) p \\
9 \pm 0.4(p= \\
\text { adjusted for }\end{array}$ & $\begin{array}{l}\text { G1. Pre (2.54 } \\
\text { G2. Pre (2.36 } \\
\text { G1 vs. G2 - } \\
=0.289 ; \text { Pow }\end{array}$ & $\begin{array}{l} \pm 0.63) \text { vs. Post }(1.82 \pm 0.42) \\
\quad p<0.001 \\
\pm 0.83) \text { vs. Post }(1.83 \pm 0.73) \\
\quad p<0.001 \\
\\
.86 \pm 0.5 \text { vs. }-0.63 \pm 0.8(p \\
=0.283 ; p=0.554 \text { adjusted } \\
\quad \text { for age }) .\end{array}$ \\
\hline & Senna (2019) & $\begin{array}{l}\text { G1. Platelet-rich } \\
\text { plasma } \\
\text { G2: } 1 \text { mL methyl- } \\
\text { prednisolone } \\
\text { acetate }\end{array}$ & $\begin{array}{c}\text { VAS } \\
\text { G1 }(\mathrm{F}=150,217 \\
p<0.001) \\
\text { Pre: } 68.1 \pm 6.0 \\
1 \text { month: } 24.4 \\
\quad \pm 7.3 \\
3 \text { months: } 21.8 \\
\quad \pm 6.5\end{array}$ & $\begin{array}{c}\mathrm{G} 2(\mathrm{~F}=116,217 ; p<0.001) \\
\text { Pre: } 69.5 \pm 4.9 \\
1 \text { month: } 25.9 \pm 8.3 \\
3 \text { months: } 25.2 \pm 7.1 \\
\end{array}$ & $\begin{array}{c}\text { G1 vs. G2 } \\
\text { Pre: } \mathrm{t}=1.178 ; p= \\
0.242 \\
1 \text { month: } \mathrm{t}= \\
0.337 ; p=0.737 \\
3 \text { months: } \mathrm{t}= \\
2.100 ; p=0.040\end{array}$ & $\begin{array}{c}\text { G1 (F= 94,739; } p<0.001) \\
\text { Pre: } 3.5 \pm 0.4 \\
1 \text { month: } 2.4 \pm 0.6 \\
3 \text { months: } 2.0 \pm 0.7 \\
\\
\text { G1 vs. G2 } \\
\text { Pre: } \mathrm{t}=1.1082 ; p \\
1 \text { month: } \mathrm{t}=0.268 ; \gamma \\
3 \text { months: } \mathrm{t}=2.752 ;\end{array}$ & $\begin{array}{c}\mathrm{G} 2(\mathrm{~F}= \\
89,111 ; p< \\
0.001) \\
\text { Pre: } 3.4 \pm \\
0.4 \\
1 \text { month: } \\
2.5 \pm 0.5 \\
3 \text { months: } \\
2.4 \pm 0.7 \\
274 \\
0.790 \\
0.007\end{array}$ & $\begin{array}{c}\mathrm{G} 1 \\
(\mathrm{~F}=111,916 ; \\
p<0.001) \\
\text { Pre: } 3.5 \pm \\
0.4 \\
1 \text { month: } \\
2.5 \pm 0.5 \\
3 \text { months: } \\
2.1 \pm 0.6 \\
\\
\text { Pre: } \\
1 \text { mon } \\
3 \text { mont }\end{array}$ & $\begin{array}{c}\mathrm{G} 2(\mathrm{~F}=71,821 ; p<0.001) \\
\text { Pre: } 3.4 \pm 0.5 \\
1 \text { month: } 3.0 \pm 0.4 \\
3 \text { months: } 2.5 \pm 0.6 \\
\\
\text { G1 vs. G2 } \\
\mathrm{t}=1.282 ; p=0.204 \\
\text { th: } \mathrm{t}=1.283 ; p=0.203 \\
\text { hs: } \mathrm{t}=1.385 ; p=0.002\end{array}$ \\
\hline
\end{tabular}


Table 4. Cont.

\begin{tabular}{|c|c|c|c|c|c|c|c|c|c|}
\hline \multirow{2}{*}{ Group } & \multirow{2}{*}{ Study } & \multirow{2}{*}{ Treatment } & \multirow{2}{*}{\multicolumn{3}{|c|}{ Outcome Pain }} & \multicolumn{4}{|c|}{ Outcome BCTQ } \\
\hline & & & & & & SSS & & & FSS \\
\hline & Wang (2017) & $\begin{array}{c}\text { G1. } \\
\text { Triamcinolone + } \\
\text { lidocaine + Splint } \\
\text { G2. } \\
\text { Triamcinolone + } \\
\text { lidocaine }\end{array}$ & $\begin{array}{c}\text { VAS. } \\
\text { G1 } \\
\text { Pre: } 3.61 \pm 3.27 \\
\text { 6 weeks: } 0.80 \pm \\
2.00 \\
12 \text { weeks: } 1.00 \\
\pm 2.05\end{array}$ & $\begin{array}{c}\mathrm{G} 2 \\
\text { Pre: } 2.23 \pm 2.59 \\
\text { 6 weeks: } 0.40 \pm 0.84 \\
\text { 12 weeks: } 0.80 \pm 1.57\end{array}$ & $\begin{array}{c}\text { G1 vs. G2 } \\
6 \text { weeks: } 0.40 \\
(-0.45 \text { to } 1.25) p= \\
0.993 \\
12 \text { weeks: } 0.19 \\
(-0.82 \text { to } 1.21) p \\
=0.898\end{array}$ & $\begin{array}{c}\text { G1 } \\
\text { Pre: } 2.27 \pm 0.71 \\
\text { 6 weeks: } 1.30 \pm 0.53 \\
\text { 12 weeks: } 1.32 \pm 0.43 \\
\text { G1 vs. G2 } \\
\text { 6 weeks: } 0.02(-0.20 \text { to } 0.2 \\
\text { 12 weeks: }-0.17(-0.43 \text { to } 0.0\end{array}$ & $\begin{array}{c}\mathrm{G} 2 \\
\text { Pre: } 1.96 \pm \\
0.62 \\
6 \text { weeks: } \\
1.28 \pm 0.21 \\
12 \text { weeks: } \\
1.49 \pm 0.51 \\
\\
p=0.216 \\
p=0.246\end{array}$ & $\begin{array}{c}\text { G1 } \\
\text { Pre: } 1.93 \pm \\
0.78 \\
6 \text { weeks: } \\
1.33 \pm 0.56 \\
12 \text { weeks: } \\
1.27 \pm 0.50 \\
\\
6 \text { weeks: } 0 \\
12 \text { weeks: }\end{array}$ & $\begin{array}{c}\text { G2 } \\
\text { Pre: } 1.61 \pm 0.62 \\
\text { 6 weeks: } 1.18 \pm 0.28 \\
12 \text { weeks: } 1.32 \pm 0.35 \\
\text { G1 vs. G2 } \\
.14(-0.10 \text { to } 0.40) p=0.654 \\
0.04(-0.29 \text { to } 0.19) p=0.134\end{array}$ \\
\hline & Wu (2017) & $\begin{array}{l}\text { G1: Perineural } \\
\text { injection of } 5 \% \\
\text { dextrose } \\
\text { G2: Perineural } \\
\text { injection with } \\
\text { saline }\end{array}$ & $\begin{array}{c}\text { VAS. } \\
\text { G1 } \\
\text { Pre: } 6.67 \pm 0.30 \\
1 \text { month: } 4.60 \\
\pm 0.35(p< \\
0.001) \\
3 \text { months: } 3.57 \\
\pm 0.30(p< \\
0.001) \\
6 \text { months: } 2.43 \\
\pm 0.30(p< \\
0.001)\end{array}$ & $\begin{array}{c}\mathrm{G} 2 \\
\text { Pre: } 6.56 \pm 0.30 \\
1 \text { month: } 5.64 \pm 0.35(p= \\
0.002) \\
3 \text { months: } 4.70 \pm 0.46(p< \\
0.001) \\
6 \text { months: } 4.59 \pm 0.46(p< \\
0.001)\end{array}$ & $\begin{array}{c}\text { G1 vs. G2 } \\
1 \text { month: }-2.07 \pm \\
0.24 \text { vs. }-0.93 \pm \\
0.21(p=0.001) \\
3 \text { months: }-3.10 \\
\pm 0.35 \text { vs. }-1.86 \\
\pm 0.37(p=02) \\
6 \text { months: }-4.23 \\
\pm 0.33 \text { vs. }-1.98 \\
\pm 0.37(p<0.001)\end{array}$ & 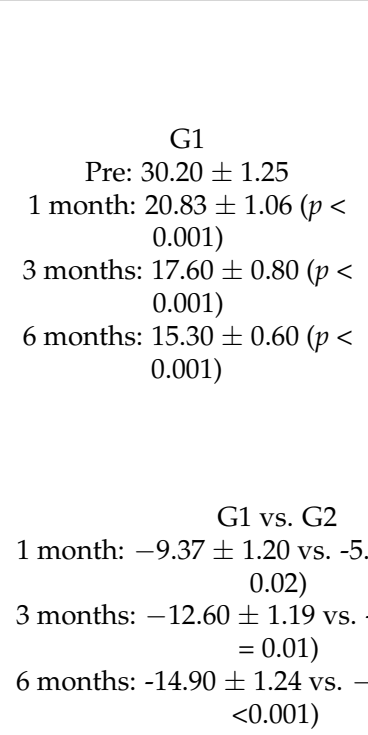 & $\begin{array}{c}\mathrm{G} 2 \\
\text { Pre: } 28.07 \\
\pm 1.93 \\
1 \text { month: } \\
22.37 \pm \\
1.76(p \\
<.001) \\
3 \text { months: } \\
20.50 \pm \\
2.02(p< \\
0.001) \\
6 \text { months: } \\
21.60 \pm \\
2.06(p= \\
0.002) \\
0 \pm 0.93(p= \\
\\
7.57 \pm 1.54(p \\
\\
5.47 \pm 1.46(p\end{array}$ & $\begin{array}{c}\mathrm{G} 1 \\
\text { Pre: } 21.87 \\
\pm 0.69 \\
1 \text { month: } \\
14.17 \pm \\
0.72(p< \\
0.001) \\
3 \text { months: } \\
12.90 \pm \\
0.52(p< \\
0.001) \\
6 \text { months: } \\
11.43 \pm \\
0.46(p< \\
0.001) \\
1 \text { month: }-7 \\
\\
3 \text { months: } \\
\\
6 \text { months: }\end{array}$ & $\begin{array}{c}\text { G2 } \\
\text { Pre: } 19.93 \pm 0.96 \\
\text { 1 month: } 18.00 \pm 1.05(p= \\
09) \\
3 \text { months: } 16.77 \pm 1.18(p \\
=0.005) \\
6 \text { months: } 17.07 \pm 1.23(p \\
=03) \\
\\
\text { G1 vs. G2 } \\
.70 \pm 0.97 \text { vs. }-1.93 \pm 0.65(p \\
<0.001) \\
-8.97 \pm 0.73 \text { vs. }-3.17 \pm 0.79 \\
(p<0.001) \\
-10.43 \pm 0.83 \text { vs. }-2.87 \pm 0.86 \\
(p<0.001)\end{array}$ \\
\hline
\end{tabular}


Table 4. Cont.

\begin{tabular}{|c|c|c|c|c|c|c|c|c|c|c|c|}
\hline \multirow{2}{*}{ Group } & \multirow{2}{*}{ Study } & \multirow{2}{*}{ Treatment } & \multirow{2}{*}{\multicolumn{3}{|c|}{ Outcome Pain }} & \multicolumn{6}{|c|}{ Outcome BCTQ } \\
\hline & & & & & & \multicolumn{3}{|c|}{ SSS } & \multicolumn{3}{|c|}{ FSS } \\
\hline & Wu (2018) & $\begin{array}{l}\text { G1. 5mL Dextrose } \\
\text { G2. 3mL } \\
\text { Triamcinolone }\end{array}$ & $\begin{array}{c}\text { VAS. } \\
\text { G1 } \\
\text { Pre: } 6.3 \pm 0.3 \\
1 \text { month: } 4.2 \pm 0.3 ; \\
-2.1(-1.4 \text { to }-2.8) \\
(p<0.001) \\
3 \text { months: } 3.3 \pm 0.2 \\
-3.1(-2.2 \text { to }-3.9) \\
(p<0.001) \\
4 \text { months: } 2.8 \pm 0.3 \\
-3.6(-2.6 \text { to }-4.5) \\
(p<0.001) \\
6 \text { months: } 2.0 \pm 0.3 \\
-4.3(-3.2 \text { to }-5.4) \\
(p<0.001)\end{array}$ & $\begin{array}{c}\text { G2 } \\
\text { Pre: } 6.2 \pm 0.2 \\
1 \text { month: } 4.2 \pm 0.4 \\
-2.1(-1.0 \text { to }-3.2) \\
(p \leq 0.001) \\
3 \text { months: } 3.57 \pm 0.30 \\
-2.6(-1.7 \text { to }-3.5) \\
(p<0.001) \\
4 \text { months: } 3.57 \pm 0.30 \\
-2.3(-1.4 \text { to }-3.3) \\
(p<0.001) \\
6 \text { months: } 2.43 \pm 0.30 \\
-1.7(-0.7 \text { to }-2.7) \\
(p<0.001)\end{array}$ & $\begin{array}{c}\text { G1 vs. G2 } \\
1 \text { month: }(p> \\
05) \\
3 \text { months: }(p> \\
05) \\
4 \text { months }(p< \\
01) \\
6 \text { months }(p> \\
001)\end{array}$ & $\begin{array}{r}\text { Pre: } 2 \\
1 \text { month } \\
-8.4(-4.5 \\
0 \\
3 \text { months } \\
-3.1(-2.2 \\
0 \\
4 \text { months } \\
-12.3(-8.4 \\
0 \\
6 \text { months } \\
-13.5 \text { (-9.3 } \\
0 .\end{array}$ & $\begin{array}{l}\text { G1 } \\
8.2 \pm 1.2 \\
: 19.8 \pm 0.9 ; \\
\text { to }-12.4)(p< \\
001) \\
: 16.4 \pm 0.7 ; \\
\text { to }-3.9)(p< \\
001) \\
: 15.9 \pm 0.6 ; \\
\text { to }-16.2)(p< \\
001) \\
: 14.7 \pm 0.6 ; \\
\text { to }-17.6)(p< \\
001)\end{array}$ & $\begin{array}{c}\mathrm{G} 2 \\
\text { Pre: } 27.6 \pm \\
1.4 \\
1 \text { month: } \\
22.5 \pm 1.7 \\
-5.0(-0.6 \\
\text { to }-9.4)(p \\
=016) \\
3 \text { months: } \\
19.8 \pm 1.2 ; \\
-7.8(-3.5 \\
\text { to }-12.0)(p \\
<0.001) \\
4 \text { months: } \\
21.2 \pm 1.3 ; \\
-6.4(-1.8 \\
\text { to }-10.9)(p \\
=0.002) \\
6 \text { months: } \\
23.7 \pm 1.6 ; \\
-3.9(0.6 \text { to } \\
-8.3)(p= \\
128)\end{array}$ & $\begin{array}{c}\text { G1 } \\
\text { Pre: } 20.7 \pm \\
1.1 \\
1 \text { month: } \\
15.0 \pm 0.8 ; \\
-5.7(-2.6 \\
\text { to }-8.9)(p \\
<0.001) \\
3 \text { months: } \\
3.3 \pm 0.2 ; \\
-3.1(-2.2 \\
\text { to }-3.9)(p \\
<0.001) \\
4 \text { months: } \\
2.8 \pm 0.3 ; \\
-3.6(-2.6 \\
\text { to }-4.5)(p \\
<0.001) \\
6 \text { months: } \\
2.0 \pm 0.3 ; \\
-4.3(-3.2 \\
\text { to }-5.4)(p \\
<0.001)\end{array}$ & $\begin{array}{r}\text { Pre: } 1 \\
1 \text { month } \\
-3.6(-0 . \\
0 \\
3 \text { month } \\
-4.7(-2 . \\
0 \\
4 \text { month } \\
-3.7(-1 . \\
0 \\
6 \text { month } \\
-3.0(1.0 \text { to }\end{array}$ & $\begin{array}{l}7 \\
7 \pm 0.8 \\
6.1 \pm 1.0 ; \\
0-6.5)(p< \\
01) \\
15.0 \pm 0.8 ; \\
o-7.2)(p< \\
01) \\
15.9 \pm 0.8 ; \\
o-6.4)(p< \\
01) \\
16.6 \pm 0.8 ; \\
6.2)(p=063)\end{array}$ \\
\hline Electrotherapy & $\begin{array}{l}\text { Çatalbas } \\
\text { (2018) }\end{array}$ & $\begin{array}{c}\text { G1. Continuous } \\
\text { ultrasound + Night } \\
\text { splint } \\
\text { G2. Pulsed } \\
\text { ultrasound + Night } \\
\text { splint } \\
\text { G3. Placebo } \\
\text { Ultrasound + Night } \\
\text { splint }\end{array}$ & $\begin{array}{c}\text { VAS. } \\
\text { G1 } \\
\text { Pre: } 5.5 \pm 3.2 \\
2 \text { weeks: } 2.8 \pm 1.9(p \\
<0167) \\
6 \text { weeks: } 2.0 \pm 2.2(p \\
<0167) \text { vs. pre and } \\
\text { post. }\end{array}$ & $\begin{array}{c}\mathrm{G} 2 \\
\text { Pre: } 5.4 \pm 2.5 \\
2 \text { weeks: } 2.9 \pm 2.4(p \\
<0167) \\
6 \text { weeks: } 2.1 \pm 2.3(p \\
<0167) \text { vs. pre. }\end{array}$ & $\begin{array}{c}\text { G3 } \\
\text { Pre: } 5.0 \pm 2.5 \\
2 \text { weeks: } 2.6 \pm \\
2.1(p<0167) \\
6 \text { weeks: } 2.2 \pm \\
2.0(p<0167) \\
\text { vs. pre. }\end{array}$ & $\begin{array}{c}\text { G1 } \\
\text { Pre: } 2.8 \pm \\
0.8 \\
2 \text { weeks: } \\
1.9 \pm 0.7(p \\
<0167) \\
6 \text { weeks: } \\
1.9 \pm 0.7(p \\
<0167) \text { vs. } \\
\text { pre. } \\
\text { Pre: } p=0.380\end{array}$ & $\begin{array}{c}\mathrm{G} 2 \\
\text { Pre: } 3.1 \pm 0.8 \\
2 \text { weeks: } 2.1 \\
\pm 0.7(p< \\
0167) \\
6 \text { weeks: } 2.1 \\
\pm 0.9(p< \\
0167) \text { vs. pre. }\end{array}$ & $\begin{array}{c}\text { G3 } \\
\text { Pre: } 2.8 \pm \\
0.9 \\
2 \text { weeks: } \\
2.2 \pm 0.8(p \\
<0167) \\
6 \text { weeks: } \\
2.2 \pm 0.7 \text { ( } p \\
<0167) \text { vs. } \\
\text { pre. } \\
0 ; 6 \text { weeks: } p\end{array}$ & $\begin{array}{c}\text { G1 } \\
\text { Pre: } 2.5 \pm \\
0.9 \\
2 \text { weeks: } \\
1.8 \pm 0.7(p \\
<0167) \\
6 \text { weeks: } \\
2.4 \pm 0.8(p \\
<0167) \text { vs. } \\
\text { pre. } \\
\text { Pre: } p=0.91(\end{array}$ & $\begin{array}{c}\text { G2 } \\
\text { Pre: } 2.4 \pm \\
0.9 \\
2 \text { weeks: } \\
1.9 \pm 0.8(p \\
<0167) \\
6 \text { weeks: } \\
1.9 \pm 0.8(p \\
<0167) \text { vs. } \\
\text { pre. } \\
2 \text { weeks: } p= \\
p=0.260\end{array}$ & $\begin{array}{c}\text { G3 } \\
\text { Pre: } 2.4 \pm \\
0.8 \\
2 \text { weeks: } \\
1.9 \pm 0.6(p \\
<0167) \\
6 \text { weeks: } \\
1.9 \pm 0.7 \text { ( } p \\
<0167) \text { vs. } \\
\text { pre. } \\
580 ; 6 \text { weeks }\end{array}$ \\
\hline
\end{tabular}


Table 4. Cont.

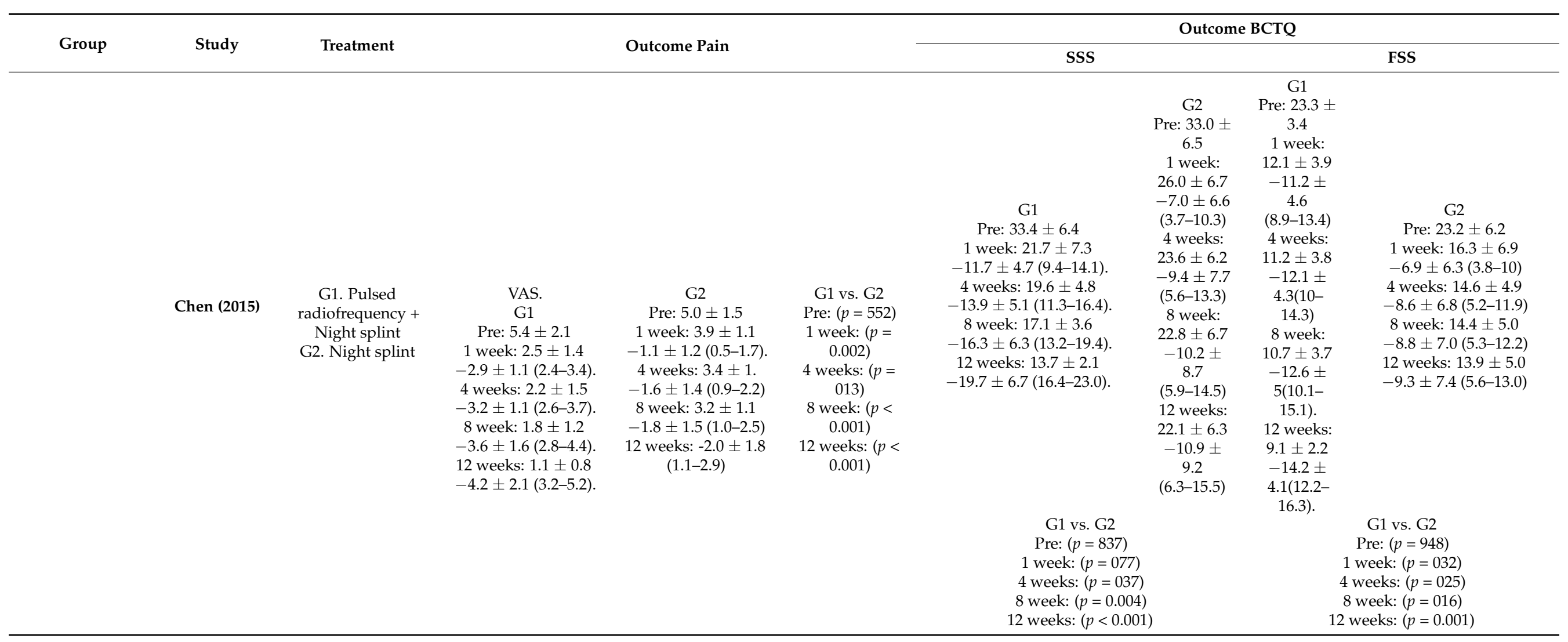


Table 4. Cont.

\begin{tabular}{|c|c|c|c|c|c|c|c|c|c|}
\hline \multirow{2}{*}{ Group } & \multirow{2}{*}{ Study } & \multirow{2}{*}{ Treatment } & \multirow{2}{*}{\multicolumn{3}{|c|}{ Outcome Pain }} & \multicolumn{4}{|c|}{ Outcome BCTQ } \\
\hline & & & & & & SSS & & & FSS \\
\hline & Chung (2016) & $\begin{array}{l}\text { G1. Electro- } \\
\text { acupuncture + } \\
\text { Night splint } \\
\text { G2. Night splint }\end{array}$ & $\begin{array}{c}\text { NPRS. } \\
\text { G1 } \\
\text { Pre: } 4.38 \pm 2.62 \\
1 \text { week: } 18(20.0) \\
-0.22(-0.68 \text { to } 0.23) \\
2 \text { weeks: } 21(23.3) \\
-0.30(-0.81 \text { to } 0.21) \\
5 \text { week: } 27(31.0) \\
-0.68(-1.18 \text { to }-0.19) \\
17 \text { weeks: } 34(40.0) \\
-1.22(-1.79 \text { to }-0.65)\end{array}$ & $\begin{array}{c}\mathrm{G} 2 \\
\text { Pre: } 4.52 \pm 2.78 \\
1 \text { week: } 20(22.0) \\
-0.43(-0.89 \text { to } 0.04) \\
2 \text { weeks: } 24(26.4) \\
-0.50(-1.01 \text { to } 0.01) \\
5 \text { week: } 28(31.1) \\
-0.55(-1.11 \text { to } 0.02) \\
17 \text { weeks: } 31(34.8) \\
-0.61(-1.22 \text { to } 0.00)\end{array}$ & $\begin{array}{c}\text { G1 vs. G2 } \\
1 \text { week: }(p=6) \\
4 \text { weeks: }(p=7) \\
8 \text { week: }(p=6) \\
12 \text { weeks: }(p= \\
03)\end{array}$ & $\begin{array}{c}\text { G1 } \\
\text { Pre: } 2.32 \pm 0.62 \\
1 \text { week: } 11(12.2) \\
0.04(-0.03 \text { to } 0.12) \\
2 \text { weeks: } 19(21.1) \\
-0.01(-0.09 \text { to } 0.07) \\
5 \text { week: } 33 \text { (37.9) } \\
0.17(-0.28 \text { to }-0.06) \\
17 \text { weeks: } 40(47.1) \\
-0.25(-0.37 \text { to }-0.12) \\
\\
\text { G1 vs. G2 } \\
1 \text { week: } 0.02(-0.09 \text { to } 0.13 \text {. } \\
4 \text { weeks: }-0.01(-0.13 \text { to } 0.1 \\
8 \text { week:- } 0.15(-0.29 \text { to }-0.01 \\
12 \text { weeks: }-0.20(-0.36 \text { to }-0 .\end{array}$ & $\begin{array}{c}\mathrm{G} 2 \\
\text { Pre: } 2.40 \pm \\
0.69 \\
1 \text { week: } 19 \\
(20.9) \\
0.01(-0.08 \\
\text { to } 0.10) \\
\text { 2 weeks: } 23 \\
(25.3) \\
-0.02(-0.13 \\
\text { to } 0.08) \\
5 \text { week: } 27 \\
(30.0) \\
-0.06(-0.19 \\
\text { to } 0.07) \\
17 \text { weeks: } \\
32(36.0) \\
-0.09(-0.25 \\
\text { to } 0.06) \\
\\
(p=8) \\
1)(p=9) \\
(p=0.04) \\
33)(p=02)\end{array}$ & $\begin{array}{c}\text { G1 } \\
\text { Pre: } 4.38 \pm \\
2.62 \\
1 \text { week: } 7 \\
(7.8) \\
0.14(0.05 \\
\text { to } 0.23) \\
\text { 2 weeks: } 15 \\
(16.7) \\
0.11(0.00 \\
\text { to } 0.22) \\
5 \text { week: } 19 \\
\text { (21.8) } \\
-0.01(-0.12 \\
\text { to } 0.11) \\
17 \text { weeks: } \\
30(35.3) \\
-0.16(-0.28 \\
\text { to }-0.04) \\
1 \text { week: } \\
4 \text { weeks: } \\
8 \text { week: } \\
12 \text { weeks: }\end{array}$ & $\begin{array}{c}\mathrm{G} 2 \\
\text { Pre: } 4.52 \pm 2.78 \\
1 \text { week: } 17(18.7) \\
0.09(0.00 \text { to } 0.18) \\
2 \text { weeks: } 15(16.5) \\
0.07(-0.04 \text { to } 0.17) \\
5 \text { week: } 18(20.0) \\
0.06(-0.07 \text { to } 0.18) \\
17 \text { weeks: } 21(23.6) \\
0.02(-0.13 \text { to } 0.17)\end{array}$ \\
\hline & $\begin{array}{c}\text { Fusakul } \\
\text { (2014) }\end{array}$ & $\begin{array}{l}\text { G1. Diode laser + } \\
\text { Splint } \\
\text { G2. Laser Placebo + } \\
\text { Splint }\end{array}$ & $\begin{array}{c}\text { VAS. } \\
\text { G1 } \\
\text { Pre: } 6.26 \pm 0.27(p< \\
0.05) \\
5 \text { weeks: } 4.25 \pm 0.34 \\
(p<0.05) \\
12 \text { weeks: } 3.45 \pm \\
0.38(p<0.05)\end{array}$ & $\begin{array}{c}\mathrm{G} 2 \\
\text { Pre: } 4.83 \pm 0.33(p< \\
0.05) \\
5 \text { weeks: } 3.15 \pm 0.30 \\
(p<0.05) \\
12 \text { weeks: } 2.48 \pm 0.36 \\
(p<0.05)\end{array}$ & $\begin{array}{c}\text { G1 vs. G2 } \\
\text { Pre: } p=0.174 \\
5 \text { weeks: } p= \\
0.243 \\
12 \text { weeks: } p= \\
0.433\end{array}$ & $\begin{array}{c}\mathrm{G} 1 \\
\text { Pre: } 2.10 \pm 0.68(p<0.05) \\
5 \text { weeks: } 1.68 \pm 0.66(p< \\
0.05) \\
12 \text { weeks: } 1.49 \pm 0.58(p< \\
0.05) \\
\\
\text { G1 vs. G2 } \\
\text { Pre: } p=0.291 \\
5 \text { weeks: } p=0.031 \\
12 \text { weeks } p=0.886\end{array}$ & $\begin{array}{c}\mathrm{G} 2 \\
\text { Pre: } 1.68 \pm \\
0.56(p< \\
0.05) \\
5 \text { weeks: } \\
1.43 \pm 0.49 \\
(p<0.05) \\
12 \text { weeks: } \\
1.35 \pm 0.51 \\
(p<0.05)\end{array}$ & $\begin{array}{c}\mathrm{G} 1 \\
\text { Pre: } 2.07 \pm \\
0.67(p< \\
0.05) \\
5 \text { weeks: } \\
1.75 \pm 0.62 \\
(p<0.05) \\
12 \text { weeks: } \\
1.53 \pm 0.57 \\
(p<0.05)\end{array}$ & $\begin{array}{c}\text { G2 } \\
\text { Pre: } 1.77 \pm 0.62(p<0.05) \\
5 \text { weeks: } 1.54 \pm 0.62(p< \\
0.05) \\
\text { 12 weeks: } 1.37 \pm 0.49(p< \\
0.05) \\
\\
\text { G1 vs. G2 } \\
\text { Pre: } p=0.712 \\
\text { weeks: } p=0.406 \\
\text { weeks: } p=0.313\end{array}$ \\
\hline
\end{tabular}


Table 4. Cont

\begin{tabular}{|c|c|c|c|c|c|c|c|c|c|c|c|c|}
\hline \multirow{2}{*}{ Group } & \multirow{2}{*}{ Study } & \multirow{2}{*}{ Treatment } & \multirow{2}{*}{\multicolumn{4}{|c|}{ Outcome Pain }} & \multicolumn{6}{|c|}{ Outcome BCTQ } \\
\hline & & & & & & & & SSS & & & FSS & \\
\hline & Güner (2018) & $\begin{array}{l}\text { G1. Low-intensity } \\
\text { laser } \\
\text { G2. Low intensity } \\
\text { laser + Kinesio-tape } \\
\text { G3. Laser Placebo }\end{array}$ & $\begin{array}{c}\text { VAS night } \\
\text { G1 } \\
\text { Post: } 8(4-10) \\
3 \text { weeks: } 2(0-8) p< \\
0.001 \\
12 \text { weeks: } 0(0-8) p< \\
0.001\end{array}$ & $\begin{array}{c}\text { G2 } \\
\text { Post: } 6 \\
(4-10) \\
3 \text { weeks: } \\
1(0-8) p \\
<0.001 \\
12 \\
\text { weeks:2 } \\
(0-6) p< \\
0.001 \\
\\
\text { G2 } \\
\text { Post: } 8 \\
(3-10) \\
3 \text { weeks: } \\
0(0-8) p \\
<0.001 \\
12 \\
\text { weeks:0 } \\
(0-6) p< \\
0.001\end{array}$ & $\begin{array}{c}\text { G3 } \\
\text { Post: } 6(2-10) \\
3 \text { weeks: } 5(1-9) p \\
<0.001 \\
12 \text { weeks:5 (0-9) } p \\
=085 \\
\\
\\
\\
\text { G3 } \\
\text { Post: } 7(5-10) \\
3 \text { weeks:5 (1-10) } \\
p=0.001 \\
12 \text { weeks: } 7(0-10) \\
p=273\end{array}$ & $\begin{array}{c}\text { G1 vs. G2 } \\
0-3 \mathrm{w}: p=879 \\
0-12 \mathrm{w}: p=879 \\
\text { G1 vs. G3 } \\
0-3 \mathrm{w}: p=0.001 \\
0-12 \mathrm{w}: p< \\
0.001 \\
\text { G2 vs. G3 } \\
0-3 \mathrm{w}: p=0.003 \\
0-12 \mathrm{w}: p< \\
0.001 \\
\text { G1 vs. G2 } \\
0-3 \mathrm{w}: p=245 \\
0-12 \mathrm{w}: p=577 \\
\text { G1 vs. G3 } \\
0-3 \mathrm{w}: p=0.004 \\
0-12 \mathrm{w}: p< \\
0.001 \\
\text { G2 vs. G3 } \\
0-3 \mathrm{w}: p=0.001 \\
0-12 \mathrm{w}: p< \\
0.001\end{array}$ & $\begin{array}{c}\mathrm{G} 1 \\
\text { Post: } 3.09 \\
(2.09-4) \\
3 \text { weeks: } \\
1.36 \\
(1.00-3.27) \\
p<0.001 \\
12 \text { weeks: } \\
1.90 \\
(1.00-3.00) \\
p<0.001\end{array}$ & $\begin{array}{c}\mathrm{G} 2 \\
\text { Post: } 3.24 \\
(2.63-4.27) \\
3 \text { weeks: } 1.54 \\
(1-2.81) p< \\
0.001 \\
12 \text { weeks: } \\
1.90(1-2.90) \\
p<0.001\end{array}$ & $\begin{array}{c}\text { G3 } \\
\text { Post: } 3.27 \\
\text { (2.09-4.18) } \\
\text { 3 weeks: } \\
2.63 \\
(1.54-3.54) \\
p=0.001 \\
12 \text { weeks: } \\
3.00 \\
(1.36-3.81) \\
p=054\end{array}$ & $\begin{array}{c}\text { G1 } \\
\text { Post: } 2.63 \\
(1.25-3.75) \\
3 \text { weeks: } \\
1.63 \\
(1.00-3.25) \\
p<0.001 \\
12 \text { weeks: } \\
1.63 \\
(1.00-3.25) \\
p<0.001\end{array}$ & $\begin{array}{c}\text { G2 } \\
\text { Post: } 2.88 \\
(1.38-3.88) \\
3 \text { weeks: } \\
1.63 \\
(1.25-2.75) \\
p<0.001 \\
12 \text { weeks: } \\
1.88(1-3) p \\
<0.001\end{array}$ & $\begin{array}{c}\text { G3 } \\
\text { Post: } 3.12 \\
\text { (1.13-5.75) } \\
\text { 3 weeks: } 3 \\
(1-5.75) p= \\
632 \\
12 \text { weeks: } \\
2.5 \text { (1-4.13) } \\
p=626\end{array}$ \\
\hline & $\begin{array}{l}\text { Kumnerddee } \\
\text { (2010) }\end{array}$ & $\begin{array}{l}\text { G1. Electro- } \\
\text { acupuncture } \\
\text { G2. Night splint }\end{array}$ & $\begin{array}{c}\text { VAS. } \\
\text { G1 } \\
\text { Post vs. Post: } \\
22.57 \pm 22.27 \text { vs. } \\
7.97+14.99\end{array}$ & $22.57 \pm 22$ & $\begin{array}{l}\text { G2 } \\
\text { st vs. Post: } \\
27 \text { vs. } 17.60+22.37\end{array}$ & $\begin{array}{c}\text { G1 vs. G2 } \\
9.63(1.07 \text { to } \\
18.20) \\
p=0.028\end{array}$ & $\begin{array}{r}\text { Post } \\
2.03 \pm 0.61 \\
\\
0.11 \text { ( }\end{array}$ & $\begin{array}{l}\text { G1 } \\
\text { vs. Post: } \\
\text { vs. } 1.98 \pm 0.56 \\
\quad \text { G1 vs. G2 } \\
-0.10 \text { to } 0.33) . p\end{array}$ & $\begin{array}{c}\text { G2 } \\
\text { Post vs. } \\
\text { Post: } \\
0.39 \text { vs. } \\
1.66 \pm 0.50 \\
0.295\end{array}$ & $\begin{array}{c}\text { G1 } \\
\text { Post vs. } \\
\text { Post: } \\
1.76 \pm 0.63 \\
\text { vs. } 1.50 \pm \\
0.39 \\
\\
0.05(-\end{array}$ & $\begin{array}{r}\text { Post v } \\
1.70 \pm 0.57 \text { v } \\
\text { G1 vs. G2 } \\
0.16 \text { to } 0.25) \cdot p\end{array}$ & $\begin{array}{l}\text { Post: } \\
1.54 \pm 0.48 \\
0.663\end{array}$ \\
\hline
\end{tabular}


Table 4. Cont.

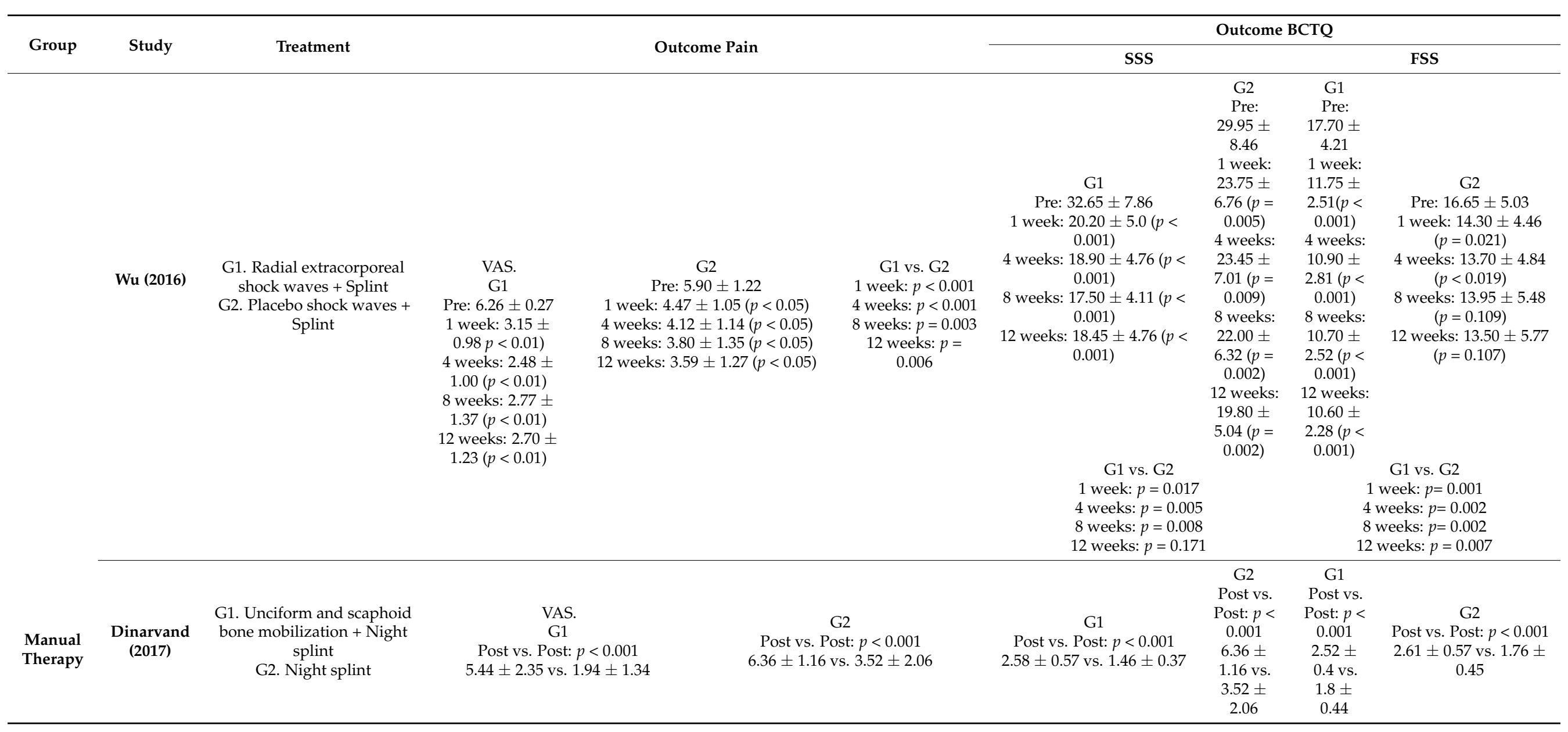


Table 4. Cont.

\begin{tabular}{|c|c|c|c|c|c|c|c|c|c|c|c|c|}
\hline \multirow{2}{*}{ Group } & \multirow{2}{*}{ Study } & \multirow{2}{*}{ Treatment } & \multirow{2}{*}{\multicolumn{4}{|c|}{ Outcome Pain }} & \multicolumn{6}{|c|}{ Outcome BCTQ } \\
\hline & & & & & & & \multicolumn{3}{|c|}{ SSS } & \multicolumn{3}{|c|}{ FSS } \\
\hline & $\begin{array}{l}\text { Geler- } \\
\text { Külcu } \\
\text { (2016) }\end{array}$ & $\begin{array}{c}\text { G1. Kinesio-tape + Gliding } \\
\text { exercises } \\
\text { G2. Placebo kinesio-tape + } \\
\text { Gliding exercises } \\
\text { G3. Night splint + Gliding } \\
\text { exercises }\end{array}$ & $\begin{array}{c}\text { VAS. } \\
\text { G1 } \\
\text { Post vs. Post: } p= \\
0.005 \\
6.6 \pm 2.1 \text { vs. } 4.1 \\
\pm 2.7 \\
\end{array}$ & $\begin{array}{c}\text { G2 } \\
\text { Post vs. Post: } \\
p=0.003 \\
5.8 \pm 3.2 \text { vs. } \\
3.9 \pm 2.8\end{array}$ & $\begin{array}{c}\text { G3 } \\
\text { Post vs. Post: } p= \\
0.024 \\
6.1 \pm 2.9 \text { vs. } 5.7 \\
\pm 3.1\end{array}$ & $\begin{array}{l}\text { G1 vs. G2 vs. G3 } \\
\quad p=0.269\end{array}$ & \multicolumn{2}{|r|}{$\begin{array}{c}\text { G2 } \\
\text { Post vs. } \\
\text { Post: } p< \\
0.0001 \\
33 \pm 10.7 \\
\text { vs. } 24.4 \pm \\
8.0\end{array}$} & $\begin{array}{c}\text { G3 } \\
\text { Post vs. } \\
\text { Post: } p< \\
0.036 \\
31.6 \pm \\
8.4 \text { vs. } \\
28.7 \pm \\
11.8 \\
.024\end{array}$ & $\begin{array}{c}\text { G1 } \\
\text { Post vs. } \\
\text { Post: } p= \\
0.001 \\
23.1 \pm \\
6.0 \mathrm{vs} . \\
16.2 \pm \\
5.4\end{array}$ & $\begin{array}{c}\text { G2 } \\
\text { Post vs. } \\
\text { Post: } p= \\
0.077 \\
19.7 \pm \\
8.4 \text { vs. } \\
16.3 \pm \\
5.8\end{array}$ & $\begin{array}{c}\text { G3 } \\
\text { Post vs. } \\
\text { Post: } p= \\
0.090 \\
19.7 \pm \\
7.0 \mathrm{vs} . \\
19.7 \pm \\
19.7\end{array}$ \\
\hline & \multirow[t]{2}{*}{$\begin{array}{l}\text { Günay } \\
\text { (2015) }\end{array}$} & \multirow[t]{2}{*}{$\begin{array}{l}\text { G1. Carpal bone } \\
\text { mobilization }+ \text { Splint } \\
\text { G2. Splint }\end{array}$} & $\begin{array}{c}\text { VAS daytime } \\
\text { G1 } \\
\text { Pre vs. Post: } p= \\
0.003 \\
3 \text { (0-8) vs. } 0(0-8)\end{array}$ & \multicolumn{2}{|c|}{$\begin{array}{c}\mathrm{G} 2 \\
\text { Pre vs. Post: } p=0.011 \\
5 \text { (0-7) vs. } 1(0-7)\end{array}$} & $\begin{array}{c}\text { G1 vs. G2 } \\
-2(-7 ; 2) \text { vs. }-3 \\
(-7 ; 4): p=0.53\end{array}$ & \multicolumn{2}{|c|}{$\begin{array}{c}\mathrm{G} 1 \\
\text { Pre vs. Post: } p<0.001 \\
29(20-46) \text { vs. } 17(12-44)\end{array}$} & $\begin{array}{c}\text { G2 } \\
\text { Pre vs. } \\
\text { Post: } p= \\
0.001 \\
31.5 \\
(18-46) \\
\text { vs. } 23 \\
(11-43)\end{array}$ & $\begin{array}{c}\text { G1 } \\
\text { Pre vs. } \\
\text { Post: } p= \\
0.001 \\
21 \\
(14-33) \\
\text { vs. } 16.5 \\
(8-32)\end{array}$ & $\begin{array}{l}\text { Pre vs. Pc } \\
19(9-35)\end{array}$ & $\begin{array}{l}t: p=0.57 \\
19(8-29)\end{array}$ \\
\hline & & & $\begin{array}{c}\text { VAS night } \\
\text { G1 } \\
\text { Pre vs. Post: } p< \\
0.001 \\
6(1-8) \text { vs. } 0(0-8)\end{array}$ & \multicolumn{2}{|c|}{$\begin{array}{c}\text { G2 } \\
\text { Pre vs. Post: } p=0.001 \\
5(0-9) \text { vs. } 0(0-8)\end{array}$} & $\begin{array}{c}\text { G1 vs. G2 } \\
-5(-8 ; 2) \text { vs. }-4 \\
(-8 ; 2): p=0.14\end{array}$ & \multicolumn{3}{|c|}{$\begin{array}{c}\text { G1 vs. G2 } \\
-12.5(-26 ; 5) \text { vs. }-8.0(-23 ; 5): p=0.39\end{array}$} & \multicolumn{3}{|c|}{$\begin{array}{l}\text { G1 vs. G2 } \\
-5.5(-18 ; 2) \text { vs. } 0(-11 ; 5): p=0.01\end{array}$} \\
\hline & $\begin{array}{l}\text { Hadianfard } \\
\quad \text { (2015) }\end{array}$ & $\begin{array}{l}\text { G1. Acupuncture }+ \text { Night } \\
\text { splint } \\
\text { G2. Night splint }+ \\
\text { Ibuprofen }\end{array}$ & $\begin{array}{c}\text { VAS. } \\
\text { G1 } \\
\text { Post vs. Post: } p< \\
0.001 \\
7.32 \pm 0.94 \text { vs. } 3.8 \\
\pm 0.78\end{array}$ & \multicolumn{2}{|c|}{$\begin{array}{c}\mathrm{G} 2 \\
\text { Post vs. Post: } p<0.001 \\
7.32 \pm 1.06 \text { vs. } 4.64 \pm 0.7\end{array}$} & $\begin{array}{c}\text { G1 vs. G2 } \\
p=0.001\end{array}$ & \multicolumn{3}{|c|}{$\begin{array}{c}\mathrm{G} 1 \\
\text { Post vs. Post: } p<0.001 \\
6.6 \pm 2.1 \text { vs. } 4.1 \pm 2.7\end{array}$} & $\begin{array}{c}\text { G1 } \\
\text { Post vs. } \\
\text { Post: } p< \\
0.001 \\
17.708 \pm \\
2.561 \mathrm{vs} . \\
11.00 \pm \\
0.780\end{array}$ & \multicolumn{2}{|c|}{$\begin{array}{c}\text { G2 } \\
\text { Post vs. Post: } p<0.001 \\
18.00 \pm 3.00 \text { vs. } 12.840 \\
\pm 1.929\end{array}$} \\
\hline
\end{tabular}


Table 4. Cont.

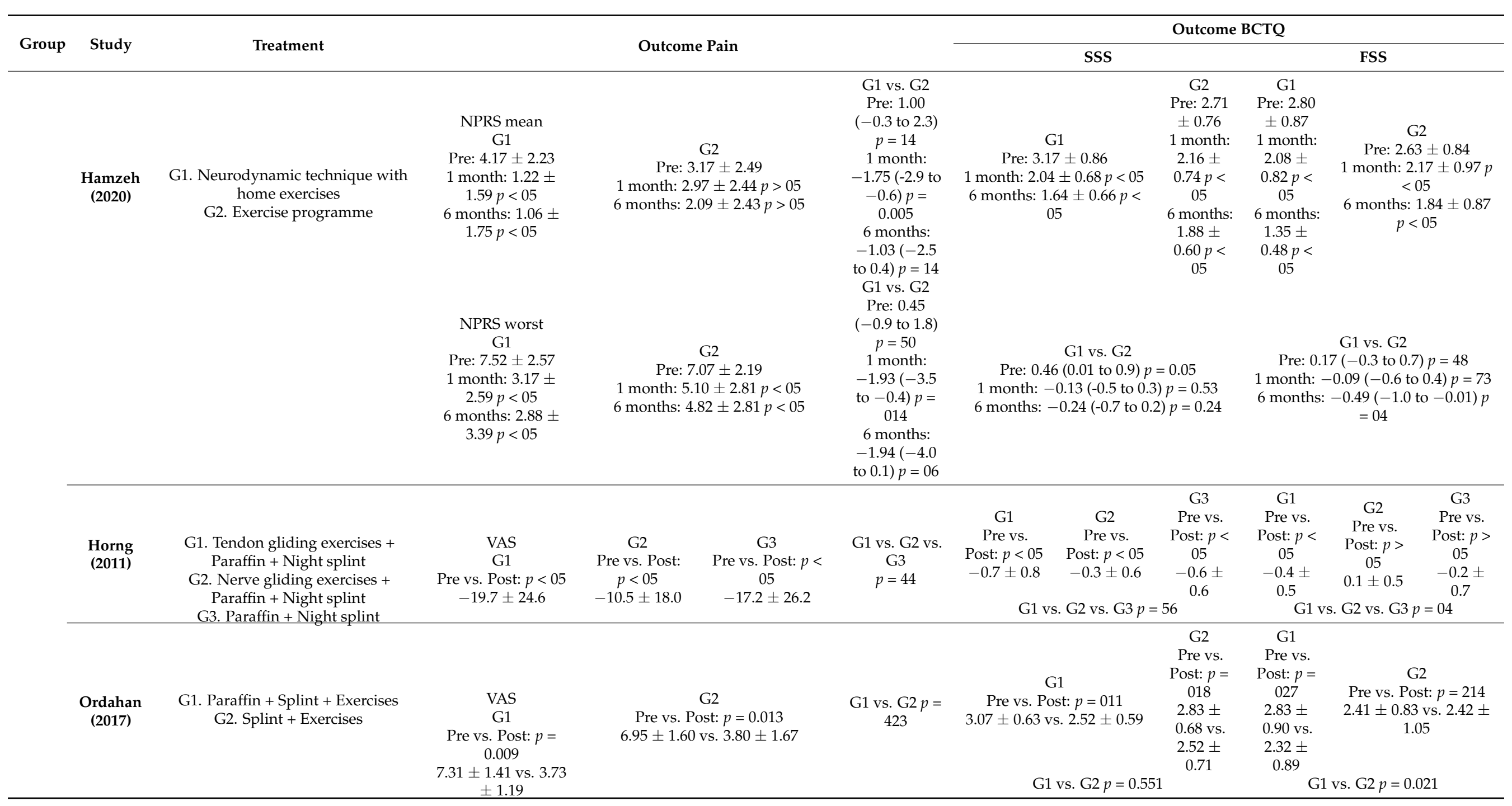


Table 4. Cont.

\begin{tabular}{|c|c|c|c|c|c|c|c|c|c|}
\hline \multirow{2}{*}{ Group } & \multirow{2}{*}{ Study } & \multirow{2}{*}{ Treatment } & \multirow{2}{*}{\multicolumn{3}{|c|}{ Outcome Pain }} & \multicolumn{4}{|c|}{ Outcome BCTQ } \\
\hline & & & & & & SSS & & & FSS \\
\hline & $\begin{array}{l}\text { Tezel } \\
\text { (2019) }\end{array}$ & $\begin{array}{l}\text { G1. Acupuncture + Night } \\
\text { splint } \\
\text { G2. Night splint }\end{array}$ & $\begin{array}{c}\text { VAS } \\
\text { G1 } \\
\text { Pre vs. Post: } \\
7.4 \pm 0.8 \text { vs. } 4.8 \\
\pm 0.8\end{array}$ & $\begin{array}{c}\text { G2 } \\
\text { Pre vs. Post: } \\
7.6 \pm 0.7 \text { vs. } 5.8 \pm 0.8\end{array}$ & $\begin{array}{c}\text { G1 vs. G2 } p= \\
0.007\end{array}$ & $\begin{array}{c}\text { G1 } \\
\text { Pre vs. Post: } \\
29.8 \pm 5.9 \text { vs. } 23.4 \pm 7.5 \\
\text { G1 vs. G2 } p=0.5\end{array}$ & $\begin{array}{l}\text { G2 } \\
\text { Pre vs. } \\
\text { Post: } \\
28.8 \pm \\
5.4 \text { vs. } \\
22.1 \pm \\
6.5\end{array}$ & $\begin{array}{l}\text { G1 } \\
\text { Pre vs. } \\
\text { Post: } \\
26.8 \pm \\
9.0 \text { vs. } \\
20.7 \pm \\
6.9 \\
\quad G\end{array}$ & $\begin{array}{c}\text { G2 } \\
\text { Pre vs. Post: } \\
25.8 \pm 8.7 \text { vs. } 19.4 \pm \\
6.4 \\
\text { vs. G2 } p=0.51\end{array}$ \\
\hline \multirow[t]{2}{*}{ Combined } & $\begin{array}{l}\text { Atthakomol } \\
\text { (2018) }\end{array}$ & $\begin{array}{l}\text { G1. Radial extracorporeal } \\
\text { shock waves } \\
\text { G2. Local corticosteroid } \\
\text { injections }\end{array}$ & $\begin{array}{c}\text { VAS } \\
\text { G1 } \\
\text { Pre: } 2.4 \pm 2.5 \\
1 \text { week: } 1.3 \pm 2.0 \\
(p<0.18) \\
4 \text { weeks: } 1.3 \pm \\
1.9(p<0.15) \\
12 \text { weeks: } 0.65 \pm \\
1.2(p<0.022) \\
24 \text { weeks: } 0.35 \pm \\
0.81(p<0.0075)\end{array}$ & $\begin{array}{c}\mathrm{G} 2 \\
\text { Pre: } 2.6 \pm 2.0 \\
\text { 1 week: } 1.6 \pm 1.7(p<0.08) \\
\text { 4 weeks: } 1.3 \pm 1.5(p<0.08) \\
\text { 12 weeks: } 1.9 \pm 2.7(p<0.52) \\
\text { 24 weeks: } 1.7 \pm 2.1(p<0.19)\end{array}$ & $\begin{array}{c}\text { G1 vs. G2 } \\
\text { Pre vs. } 1 \mathrm{w}:-10 \\
(-1.7 \text { to } 1.5) p= \\
90 \\
1 \mathrm{w} \text { vs. } 4 \mathrm{w}: 0.049 \\
(-1.6 \text { to } 1.7) p= \\
95 \\
4 \mathrm{w} \text { vs. } 12 \mathrm{w}:-1.0 \\
(-2.7 \text { to } 0.63) p= \\
23 \\
12 \mathrm{w} \text { vs. } 24 \mathrm{w}: \\
-1.2(-2.9 \text { to } \\
0.49) p=17\end{array}$ & $\begin{array}{c}\mathrm{G} 1 \\
\text { Pre: } 21 \pm 6.4 \\
1 \text { week: } 19 \pm 7.4(p=33) \\
4 \text { weeks: } 17 \pm 4.3 p=031) \\
12 \text { weeks: } 15 \pm 4.5(p= \\
0.0082) \\
24 \text { weeks: } 13 \pm 2.9(p= \\
0.0059) \\
\\
\text { G1 vs. G2 } \\
\text { Pre vs. } 1 \text { w: } 2.6(-2.0 \text { to } 7 \\
1 \text { w vs. } 4 \mathrm{w}: 1.5(-3.2 \text { to } 6 \\
\text { 4w vs. } 12 \mathrm{w}:-1.9(-2.7 \text { to } \\
\text { 12w vs. } 24 \mathrm{w}:-5.1(-9.8 \mathrm{t} \\
036\end{array}$ & $\begin{array}{c}\text { G2 } \\
\text { Pre: } 22 \pm \\
5.1 \\
1 \text { week: } \\
17 \pm 4.5 \\
(p= \\
0.0047) \\
4 \text { weeks: } \\
17 \pm 5.1 \\
(p=011) \\
12 \text { weeks: } \\
18 \pm 5.5 \\
(p=13) \\
24 \text { weeks: } \\
19 \pm 7.9 \\
(p=20) \\
\\
\\
2) p=27 \\
p=53 \\
\text {.8) } p=43 \\
-33) p= \\
\end{array}$ & $\begin{array}{c}\text { G1 } \\
\text { Pre: } 14 \pm \\
3.2 \\
1 \text { week: } \\
13 \pm 4.2 \\
(p=27) \\
4 \text { weeks: } \\
13 \pm 3.5 \\
(p=12) \\
12 \text { weeks: } \\
11 \pm 3.0 \\
(p= \\
0.0065) \\
24 \text { weeks: } \\
11 \pm 2.2 \\
(p= \\
0.0073) \\
\\
\text { Pre vs. } 1 \mathrm{w} \\
1 \mathrm{w} \text { vs. } 4 \mathrm{w} \\
4 \mathrm{w} \text { vs. } 12 \\
\\
12 \mathrm{w} \text { vs. } 2\end{array}$ & 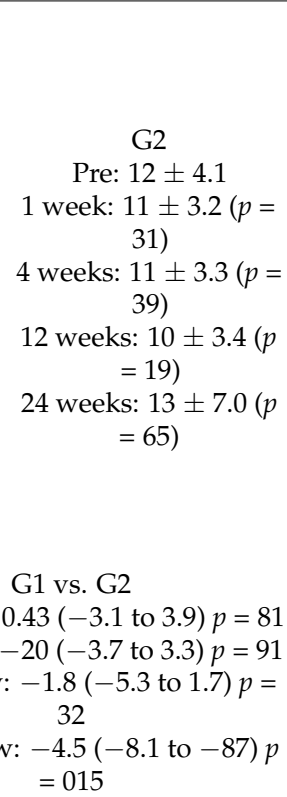 \\
\hline & $\begin{array}{c}\text { Incebiyik } \\
\text { (2015) }\end{array}$ & $\begin{array}{c}\text { G1. Short wave diathermy + } \\
\text { Gliding exercises } \\
\text { G2. Placebo + Gliding } \\
\text { exercises }\end{array}$ & $\begin{array}{c}\text { VAS } \\
\text { G1 } \\
\text { Pre vs. Post: } p< \\
0.001 \\
5.50 \pm 2.53 \text { vs. } \\
2.32 \pm 1.80\end{array}$ & $\begin{array}{c}\text { G2 } \\
\text { Pre vs. Post: } p=1.105 \\
4.83 \pm 2.76 \text { vs. } 4.20 \pm 2.53\end{array}$ & $\begin{array}{c}\text { G1 vs. G2 } p= \\
0.003\end{array}$ & $\begin{array}{c}\text { G1 } \\
\text { Pre vs. Post: } p<0.001 \\
30.78 \pm 7.92 \text { vs. } 18.53 \pm \\
9.09 \\
\\
\text { G1 vs. G2 } \mathrm{p}=0.0\end{array}$ & $\begin{array}{c}\text { G2 } \\
\text { Pre vs. } \\
\text { Post: } p= \\
204 \\
29.25 \pm \\
11.41 \text { vs. } \\
27.62 \pm \\
10.63\end{array}$ & $\begin{array}{c}\text { G1 } \\
\text { Pre vs. } \\
\text { Post: } p< \\
0.001 \\
29.8 \pm \\
5.9 \text { vs. } \\
23.4 \pm \\
7.5 \\
\text { G1 }\end{array}$ & $\begin{array}{c}\text { G2 } \\
\text { Pre vs. Post: } p=234 \\
28.8 \pm 5.4 \text { vs. } 22.1 \pm \\
6.5 \\
\\
\text { vs. G2 } p<0.001\end{array}$ \\
\hline
\end{tabular}


Table 4. Cont.

\begin{tabular}{|c|c|c|c|c|c|c|c|c|c|}
\hline \multirow{2}{*}{ Group } & \multirow{2}{*}{ Study } & \multirow{2}{*}{ Treatment } & \multirow{2}{*}{\multicolumn{3}{|c|}{ Outcome Pain }} & \multicolumn{4}{|c|}{ Outcome BCTQ } \\
\hline & & & & & & \multicolumn{2}{|l|}{ SSS } & \multicolumn{2}{|r|}{ FSS } \\
\hline & Talebi (2018) & $\begin{array}{l}\text { G1: Manual } \\
\text { therapy-Nerve + } \\
\text { interface } \\
\text { mobilization + } \\
\text { neural } \\
\text { G2: TENS + }\end{array}$ & $\begin{array}{c}\text { VAS } \\
\text { G1 } \\
\text { Pre vs. Post: } p=0.000 \\
7.08 \pm 1.56 \text { vs. } 3.75 \pm 2.22\end{array}$ & $\begin{array}{c}\mathrm{G} 2 \\
\text { Pre vs. Post: } p=0.000 \\
6.58 \pm 1.37 \text { vs. } 4.41 \pm 1.31\end{array}$ & G1 vs. G2 $p=141$ & $\begin{array}{c}\text { G1 } \\
\text { Pre vs. Post: } p=0.000 \\
29.91 \pm 9.65 \text { vs. } 19.25 \\
\pm 6.25 \\
\\
\text { G1 vs. G2 } p=0\end{array}$ & $\begin{array}{c}\text { G2 } \\
\text { Pre vs. } \\
\text { Post: } p= \\
0.000 \\
29.91 \pm \\
7.24 \text { vs. } \\
25.41 \pm \\
6.25 \\
06\end{array}$ & $\begin{array}{c}\text { G1 } \\
\text { Pre vs. } \\
\text { Post: } p= \\
241 \\
4.83 \pm \\
2.76 \text { vs. } \\
4.20 \pm \\
2.53\end{array}$ & $\begin{array}{c}\text { G2 } \\
\text { Pre vs. Post: } p=0.008 \\
4.83 \pm 2.76 \text { vs. } 4.20 \pm \\
2.53 \\
\\
\text { vs. G2 } p=043\end{array}$ \\
\hline & Wolny (2017) & $\begin{array}{c}\text { G1: Neurodynamic } \\
\text { techniques + } \\
\text { Function massage + } \\
\text { Joint mobilization } \\
\text { G2: Laser + }\end{array}$ & $\begin{array}{c}\text { NPRS } \\
\text { G1 } \\
\text { Pre vs. Post: } p<01 \\
5.72 \pm 1.49 \text { vs. } 1.47 \pm 1.20\end{array}$ & $\begin{array}{c}\text { G2 } \\
\text { Pre vs. Post: } p<01 \\
5.25 \pm 1.75 \text { vs. } 3.58 \pm 1.93\end{array}$ & G1 vs. G2 $p<01$ & $\begin{array}{c}\text { G1 } \\
\text { Pre vs. Post: } \\
2.97 \pm 0.63 \text { vs. } 1.78 \pm \\
0.47 \\
\\
\text { G1 vs. G2 } p<0\end{array}$ & $\begin{array}{c}\text { G2 } \\
\text { Pre vs. } \\
\text { Post: } \\
2.94 \pm \\
0.74 \text { vs. } \\
2.57 \pm \\
0.77 \\
01\end{array}$ & $\begin{array}{c}\text { G1 } \\
\text { Pre vs. } \\
\text { Post: } \\
2.80 \pm \\
0.94 \text { vs. } \\
1.90 \pm \\
0.62\end{array}$ & $\begin{array}{c}\text { G2 } \\
\text { Pre vs. Post: } \\
2.77 \pm 0.94 \text { vs. } 2.55 \pm \\
0.95 \\
\\
\text { vs. G2 } p<0.001\end{array}$ \\
\hline & Wolny (2019) & $\begin{array}{l}\text { G1: Neurodynamic } \\
\text { techniques } \\
\text { G2: No treatment }\end{array}$ & $\begin{array}{c}\text { NPRS } \\
\text { G1 } \\
\text { Pre vs. Post: } \\
5.86 \pm 1.46 \text { vs. } 1.38 \pm 0.72\end{array}$ & $\begin{array}{c}\text { G2 } \\
\text { Pre vs. Post: } \\
5.71 \pm 1.34 \text { vs. } 5.46 \pm 1.05\end{array}$ & $\begin{array}{c}\text { G1 vs. G2 } p< \\
0.001\end{array}$ & $\begin{array}{c}\text { G1 } \\
\text { Pre vs. Post: } \\
3.03 \pm 0.65 \text { vs. } 1.08 \pm \\
0.68 \\
\\
\text { G1 vs. } \mathrm{G} 2 \mathrm{p}<\mathrm{c}\end{array}$ & $\begin{array}{c}\text { G2 } \\
\text { Pre vs. } \\
\text { Post: } \\
2.92 \pm \\
0.71 \text { vs. } \\
2.87 \pm \\
0.68 \\
01\end{array}$ & $\begin{array}{c}\text { G1 } \\
\text { Pre vs. } \\
\text { Post: } \\
2.82 \pm \\
0.71 \text { vs. } \\
1.96 \pm \\
0.64\end{array}$ & $\begin{array}{c}\text { G2 } \\
\text { Pre vs. Post: } \\
2.99 \pm 0.67 \text { vs. } 2.87 \pm \\
0.71 \\
\\
\text { vs. G2 } p<0.001\end{array}$ \\
\hline & Xu (2020) & $\begin{array}{c}\text { G1: Radial } \\
\text { extracorporeal } \\
\text { shock waves } \\
\text { G2: Betamethasone } \\
1 \mathrm{~mL}\end{array}$ & $\begin{array}{c}\text { VAS } \\
\text { G1 } \\
\text { Pre: } 2.5 \pm 0.3 \\
\text { 3 week: } 1.4 \pm 0.9(p=0.04) \\
\text { 9 weeks: } 8 \pm 1.1(p=0.02) \\
12 \text { weeks: } 0.6 \pm 0.7(p=0.00)\end{array}$ & $\begin{array}{c}\mathrm{G} 2 \\
\text { Pre: } 2.6 \pm 0.4 \\
3 \text { week: } 1.5 \pm 1.1(p=0.04) \\
9 \text { weeks: } 1.7 \pm 0.7(p=0.21) \\
12 \text { weeks: } 1.9 \pm 1.3(p=0.17)\end{array}$ & $\begin{array}{c}\text { G1 vs. G2 } \\
3 \text { week: } p=56 \\
9 \text { weeks: } p=0.00 \\
12 \text { weeks } p=0.00\end{array}$ & $\begin{array}{r}\text { G1 } \\
\text { Pre: } 34.1 \pm 4 \\
\text { 3 week: } 30.2 \pm 3.7 \\
\text { 9 weeks: } 25.4 \pm 4.1 \\
\text { 12 weeks: } 22.3 \pm 2.7\end{array}$ & $\begin{array}{l} \\
=0.04) \\
=0.01) \\
=0.00)\end{array}$ & $\begin{array}{l}3 \text { wee } \\
9 \text { wee } \\
12 \text { we }\end{array}$ & $\begin{array}{l}\mathrm{G} 2 \\
\text { re: } 34.7 \pm 5.6 \\
28.1 \pm 6.7(p=0.03) \\
28.9 \pm 6.8(p=0.05) \\
31.8 \pm 3.4(p=53)\end{array}$ \\
\hline
\end{tabular}


Table 4. Cont.

\begin{tabular}{|c|c|c|c|c|c|c|c|c|c|c|c|c|}
\hline \multirow{3}{*}{ Group } & \multirow{2}{*}{ Study } & \multirow{2}{*}{ Treatment } & \multirow{2}{*}{\multicolumn{4}{|c|}{ Outcome Pain }} & \multicolumn{6}{|c|}{ Outcome BCTQ } \\
\hline & & & & & & & \multicolumn{3}{|c|}{ SSS } & \multicolumn{3}{|c|}{ FSS } \\
\hline & Yildiz (2011) & $\begin{array}{l}\text { G1: Ultrasound + Gel } \\
\text { with ketoprofen }+ \\
\text { Splint } \\
\text { G2: Ultrasound + Gel } \\
\text { with drugs }+ \text { Splint } \\
\text { G3: Placebo ultrasound } \\
\quad+\text { Splint }\end{array}$ & $\begin{array}{c}\text { VAS } \\
\text { G1 } \\
\text { Pre: } 5.76 \pm 2.45 \\
\text { 2weeks: } 2.72 \pm 2.07 \\
\text { 8 weeks: } 3.48 \pm 2.74\end{array}$ & $\begin{array}{c}\text { G2 } \\
\text { Pre: } 4.96 \pm \\
2.50 \\
\text { 2weeks: } 2.41 \\
\quad \pm 2.43 \\
\text { 8 weeks: } 2.77 \\
\quad \pm 2.74\end{array}$ & $\begin{array}{c}\text { G3 } \\
\text { Pre: } 2.5 \pm 0.3 \\
\text { 2weeks: } 3.03 \pm \\
1.96 \\
\text { 8 weeks: } 0.98 \pm \\
1.65\end{array}$ & $\begin{array}{l}\mathrm{G} 1<\mathrm{G} 3 p=0.002 \\
\mathrm{G} 2<\mathrm{G} 3 p=0.004\end{array}$ & $\begin{array}{c}\text { G1 } \\
\text { Pre: } 2.88 \\
\pm 0.55 \\
\text { 2weeks: } \\
1.94 \pm \\
0.57 \\
8 \text { weeks: } \\
2.08 \pm \\
0.82\end{array}$ & $\begin{array}{c}\mathrm{G} 2 \\
\text { Pre: } 2.96 \\
\pm 0.62 \\
2 \text { weeks: } \\
2.04 \pm \\
0.61 \\
8 \text { weeks: } \\
1.97 \pm \\
0.65\end{array}$ & $\begin{array}{c}\text { G3 } \\
\text { Pre: } 2.93 \\
\pm 1.04 \\
\text { 2weeks: } \\
1.78 \pm \\
0.75 \\
8 \text { weeks: } \\
1.63 \pm \\
0.73\end{array}$ & $\begin{array}{c}\text { G1 } \\
\text { Pre: } 2.73 \\
\pm 0.73 \\
\text { 2weeks: } \\
2.08 \pm \\
0.78 \\
8 \text { weeks: } \\
2.19 \pm \\
0.89\end{array}$ & $\begin{array}{c}\text { G2 } \\
\text { Pre: } 2.56 \\
\pm 0.64 \\
2 \text { weeks: } \\
1.93 \pm \\
0.55 \\
8 \text { weeks: } \\
1.98 \pm \\
0.78\end{array}$ & $\begin{array}{l}\text { G3 } \\
\text { Pre: } 2.79 \\
\pm 1.05 \\
\text { 2weeks: } \\
2.16 \pm \\
0.80 \\
8 \text { weeks: } \\
1.79 \pm \\
0.80\end{array}$ \\
\hline Other & $\begin{array}{l}\text { Eftekharsadat } \\
\text { (2018) }\end{array}$ & $\begin{array}{l}\text { G1: Lavender ointment } \\
\text { + Night splint } \\
\text { G2: Placebo ointment + } \\
\text { Night splint }\end{array}$ & $\begin{array}{c}\text { VAS } \\
\text { G1 } \\
\text { Pre vs. Post: } p=049 \\
6.96 \pm 1.30 \text { vs. } 3.58 \pm \\
1.59\end{array}$ & \multicolumn{2}{|c|}{$\begin{array}{c}\text { G2 } \\
\text { Pre vs. Post: } \\
6.12 \pm 1.54 \text { vs. } 4.79 \pm 2.36\end{array}$} & $\begin{array}{c}\text { G1 vs. G2 } p< \\
0.001\end{array}$ & $\begin{array}{r}\text { Pre } \\
2.20 \pm\end{array}$ & $\begin{array}{c}\mathrm{G} 1 \\
\text { s. Post: } p< \\
0.48 \text { vs. } 1.5 \mathrm{c}\end{array}$ & $\begin{array}{l}001 \\
\pm 0.29 \\
\text { G1 vs. G }\end{array}$ & \multicolumn{3}{|c|}{$\begin{aligned} & \text { Pre vs. Post: } p<0.001 \\
& 2.18 \pm 0.49 \text { vs. } 1.78 \pm 0.53 \\
= & 0.003\end{aligned}$} \\
\hline
\end{tabular}

BCTQ: Boston Carpal Tunnel Questionnaire; BCTQ-SSS: BCTQ Symptom Severity Scale; BCTQ-FSS: BCTQ Function Severity Scale; NPRS: Numeric Pain Rating Scale; VAS: visual analogue scale. 
Regarding pharmacological treatment, the studies compared a type of corticosteroids with other drugs or with placebos. There were no statistically significant conclusions as to their effectiveness. The studies that analyzed using platelet-rich plasma did not reveal significant differences $[33,34]$. Combining corticosteroids and night splints presented statistically significant improvements compared with using solely drugs or night splints. Electrotherapy presented statistically significant improvements over corticosteroids [47]. The most often used drugs were triamcinolone, lidocaine and methylprednisolone. One dose of $5 \%$ dextrose was statistically significant compared to placebo or triamcinolone.

Concerning electrotherapy, all the outcomes showed statistically significant improvements in pain and/or function (BCQT) against placebo or control groups. Most of these studies added a night splint as part of the treatment. In isolation, electrotherapy was not as effective compared with the control and/or placebo groups. The therapies used were shock waves [21,24,47], ultrasound [37,48], laser [38,39], electro-acupuncture [25,41], diathermy [40] and radiofrequency [22].

As far as manual therapy is concerned, the articles presented significantly better improvements in these groups in functional variables (BCQT), and some, in symptoms and strength. These studies were the main ones obtaining changes in the electrophysiological parameters. The studies that compared manual therapy and electrotherapy found significant differences in favor of the manual therapy group $[23,30]$. The interventions featured the use of neurodynamic techniques [26,27,45], acupuncture [28,44], exercise [50], joint mobilization $[43,46]$ or a combination of various techniques [23,30,42]. Nerve mobilization or neurodynamic techniques were described differently among the studies. Three studies included mobilization from shoulder to fingers, described by Shacklock [23,30,45] as initial position; shoulder abduction to $90^{\circ}$; arm external rotation; wrist and fingers extension; forearm supination; and elbow extension, compared to those who performed only wrist and hand mobilization $[27,40]$ in six different positions as grasping, finger extension, wrist extension, thumb extension, forearm supination, and gentle stretch of the thumb with the opposite hand. Joint mobilization consisted in performing distraction and dorsal/palmar glides on the first row of the radiocarpal joints [43] or dorsal and palmar glide on scaphoid and hamate bones [46]. Both studies aimed to improve wrist range of motion on CTS patients. In addition, treatment of soft tissue was complementary to other manual techniques. Acupuncture techniques were described in isolation. Both studies followed a similar protocol, involving described acupoints unilaterally for the involved side. Hadianfard et al. [44] include nine acupoints compared to Tezel et al. [28] that performed the therapy on six acupoints. Functional massage (a combination of muscle compression while mobilizing the joint) of the soft tissue was performed in two studies, complementary to other manual therapy treatment. Talebi et al. [23] performed it on pronator teres muscle, unlike Wolny et al. [30] who performed it on the trapezius muscle. Both techniques were not described.

Lastly, lavender ointment combined with a night splint did not yield any significant differences compared with the placebo groups [49]. Lavender ointment and placebo were instructed to be applied on their affected wrist in the morning and evening time for 40 days.

\subsubsection{Variables}

All the studies included assessed function using the BCTQ scale, and 3 added the DASH questionnaire on shoulder, elbow and hand impairment [25-27]. All the articles evaluated pain, with 25 using a visual analogic scale (VAS) and 4 using the numeric pain rating scale (NPRS) $[25,26,43,45]$. In addition to the evaluation of pain, 3 articles used a VAS to quantify paresthesia $[21,34,37]$.

Twenty articles analyzed electrophysiological parameters, 12 articles analyzed grip strength and/or pinch strength $[21,22,25,26,32,37,39,42,43,45]$ and 6 articles analyzed the cross sectional area of the carpal tunnel using a ultrasound-guided procedure $[21,22,29,34,36,37]$. Other variables measured were quality of life (Notting- 
ham Health Profile [NHP] and World Health Organization Quality of Life [WHOQOL]), neurodynamic tests, range of movement, or clinical parameters (Tinel and Phalen tests).

\subsubsection{Follow-Up}

Most studies had a follow-up of no more than 6 months, with the majority of the studies having short- and mid-term follow-ups. There were 24 articles that studied the short-term ( $\leq 3$ months) effects, while 5 had mid-term ( $\leq 6$ months) follow-ups. The studies analyzing drug application mainly analyzed mild term follow-ups.

\subsubsection{Evaluation of the Risk of Bias}

The methodological quality scores ranged from 6 to 10. The mean quality of all the studies analyzed by the PEDro scale [19] was 7.5 over 11.14 of the 29 studies obtained a score of 8 or more on this scale (Table 5). The blinding of the participants of the samples, the blinding of the researcher who carried out the therapy were the criteria with no achievement throughout the studies. Moreover, the criterion of "all subject for whom outcome were available received the treatment or control condition as allocated or, where this was not the case, data for at least one key outcome was analyzed by intention to treat" was less achieve by the studies.

Table 5. PEDro Scale.

\begin{tabular}{|c|c|c|c|c|c|c|c|c|c|c|c|c|}
\hline Study & 1 & 2 & 3 & 4 & 5 & 6 & 7 & 8 & 9 & 10 & 11 & Total \\
\hline $\begin{array}{l}\text { Atthakomol } \\
\text { (2018) }\end{array}$ & $x$ & $x$ & $x$ & $x$ & & & $x$ & & & $x$ & $x$ & 7 \\
\hline Çatalbas (2018) & $\mathrm{x}$ & $\mathrm{X}$ & & $\mathrm{X}$ & $x$ & & $x$ & $x$ & & $x$ & & 7 \\
\hline Chen (2015) & $\mathrm{x}$ & $\mathrm{X}$ & $\mathrm{x}$ & $\mathrm{X}$ & & & $x$ & & & $x$ & $\mathrm{X}$ & 7 \\
\hline Chung (2016) & $\mathrm{x}$ & $\mathrm{X}$ & $\mathrm{X}$ & $\mathrm{X}$ & $x$ & & $x$ & $\mathrm{x}$ & $\mathrm{X}$ & $x$ & $x$ & 10 \\
\hline Dinarvand (2017) & $x$ & $x$ & & $x$ & & & $X$ & $x$ & & $X$ & & 6 \\
\hline $\begin{array}{c}\text { Eftekharsadat } \\
(2018)\end{array}$ & $x$ & $x$ & & $x$ & $x$ & $X$ & $x$ & $x$ & & $x$ & & 8 \\
\hline Fusakul (2014) & $\mathrm{x}$ & $x$ & $x$ & $\mathrm{X}$ & $x$ & & $x$ & $\mathrm{X}$ & & $x$ & & 8 \\
\hline $\begin{array}{l}\text { Geler-Külcü } \\
\text { (2016) }\end{array}$ & $x$ & $x$ & $x$ & $x$ & $x$ & & $x$ & $x$ & & $x$ & & 8 \\
\hline Günay (2015) & $x$ & $x$ & $x$ & $x$ & & & $x$ & & & $x$ & $\mathrm{X}$ & 7 \\
\hline Güner (2018) & $x$ & $x$ & & $x$ & $x$ & & $x$ & $\mathrm{x}$ & & $\mathrm{X}$ & & 7 \\
\hline Hadianfard (2015) & $\mathrm{x}$ & $\mathrm{X}$ & & $\mathrm{X}$ & & & $x$ & $\mathrm{x}$ & $\mathrm{X}$ & $\mathrm{X}$ & $\mathrm{X}$ & 8 \\
\hline Hamzeh (2020) & $x$ & $x$ & $\mathrm{X}$ & $x$ & & & $X$ & & & $X$ & $x$ & 7 \\
\hline Horng (2011) & $\mathrm{x}$ & $\mathrm{X}$ & $\mathrm{X}$ & $\mathrm{X}$ & & & $x$ & & & $\mathrm{X}$ & & 6 \\
\hline Incebiyik (2015) & $x$ & $x$ & $x$ & $x$ & $x$ & & $x$ & & & $x$ & $x$ & 8 \\
\hline $\begin{array}{c}\text { Karimzadeh } \\
(2019)\end{array}$ & $x$ & $x$ & & $x$ & $x$ & & & $x$ & & $x$ & & 7 \\
\hline $\begin{array}{c}\text { Kumnerddee } \\
(2010)\end{array}$ & $x$ & $x$ & & $x$ & & & & $\mathrm{x}$ & & $x$ & $x$ & 6 \\
\hline Ordahan (2017) & $\mathrm{x}$ & $\mathrm{X}$ & & $\mathrm{X}$ & & & $\mathrm{X}$ & $x$ & & $x$ & & 6 \\
\hline Raeissadat (2018) & $\mathrm{x}$ & $\mathrm{X}$ & $\mathrm{X}$ & $\mathrm{X}$ & & & & $\mathrm{x}$ & $\mathrm{X}$ & $\mathrm{X}$ & $x$ & 8 \\
\hline Senna (2019) & $\mathrm{x}$ & $X$ & $\mathrm{X}$ & $\mathrm{X}$ & $x$ & & & $\mathrm{x}$ & & $x$ & $x$ & 8 \\
\hline Talebi (2018) & $\mathrm{x}$ & $\mathrm{X}$ & & $\mathrm{X}$ & & & $x$ & $\mathrm{x}$ & $\mathrm{X}$ & $\mathrm{X}$ & $x$ & 8 \\
\hline Tezel (2019) & $\mathrm{x}$ & $\mathrm{X}$ & & $\mathrm{X}$ & & & $X$ & & & $\mathrm{X}$ & $\mathrm{X}$ & 6 \\
\hline Wang (2017) & $x$ & $\mathrm{X}$ & $\mathrm{X}$ & $\mathrm{x}$ & & & $x$ & $\mathrm{x}$ & $\mathrm{X}$ & $x$ & $x$ & 9 \\
\hline Wolny (2019) & $\mathrm{x}$ & $\mathrm{X}$ & $\mathrm{x}$ & $\mathrm{X}$ & & & $x$ & & & $x$ & & 6 \\
\hline Wolny (2017) & $x$ & $x$ & $x$ & $x$ & & & $X$ & & & $x$ & $X$ & 7 \\
\hline $\mathrm{Wu}(2018)$ & $x$ & $x$ & & $x$ & & & $X$ & $\mathrm{x}$ & $\mathrm{X}$ & $\mathrm{X}$ & $\mathrm{X}$ & 8 \\
\hline $\mathrm{Wu}(2017)$ & $x$ & $x$ & $\mathrm{x}$ & $x$ & & & $X$ & $x$ & $x$ & $x$ & $X$ & 9 \\
\hline $\mathrm{Wu}(2016)$ & $\mathrm{X}$ & $\mathrm{X}$ & $\mathrm{X}$ & $\mathrm{X}$ & $x$ & & $x$ & $\mathrm{x}$ & $\mathrm{X}$ & $x$ & & 9 \\
\hline Xu (2020) & $\mathrm{x}$ & $X$ & $\mathrm{X}$ & $\mathrm{X}$ & & & $X$ & $\mathrm{X}$ & $X$ & $X$ & & 8 \\
\hline Yildiz (2011) & $x$ & $X$ & $x$ & $x$ & $x$ & & $x$ & & $x$ & $x$ & & 8 \\
\hline Mean & & & & & & & & & & & & 7.5 \\
\hline
\end{tabular}

The RoB2 tools [20] showed that the aspects with the worst methodological quality in all the studies are found in the effect of assigning to the intervention and the results reported. Variable measurement seems to have the best methodical quality in all the studies 
considered (Figure 2a,b). Even though, it seems that good methodological quality was performed in the majority of studies.

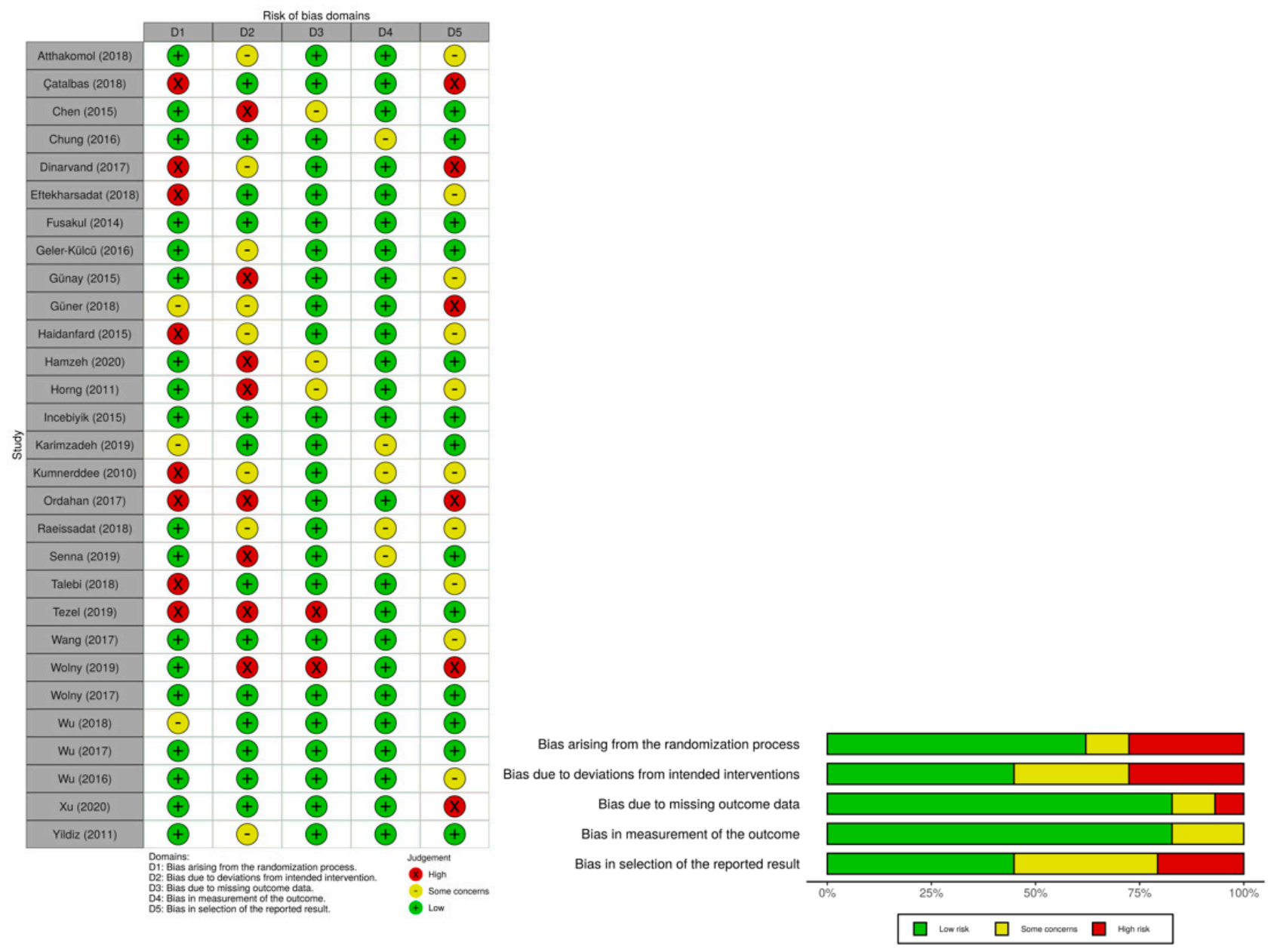

(a)

(b)

Figure 2. (a) Summary of Risk of Bias 2.0. (b) Risk of bias 2.0. graph.

\section{Discussion}

Our study objective was to review the effectiveness of conservative treatment in patients with CTS of any level of severity, with the inclusion of patients with systemic conditions. In patients having mild or moderate intensity, three types of treatment were used: drug, electrotherapy and manual therapy. Severe patients were not analyzed isolated, not allowing which kind of treatment is effective for them. Patients with systemic disease were poorly represent in samples. Two studies include patients with diabetes and hypertension, and another analyzed aa sample of diabetic patients. Manual therapy could be effective for those patients at short-term, but more future studies have to be done.

Splint therapy was the more used control or complementary intervention. Adding a wrist splint to all kind of intervention treatments seem to be more beneficial than not including the orthosis. Hall et al. [51] observed that conservative treatment program including full-time splinting and formal education can improve symptoms and function on CTS patients. It could be considered for symptom reduction in all patients waiting for the surgical intervention. Burke et al. [52] observed the angle of the splint does make a difference in subjective relief of the symptoms of carpal tunnel syndrome, and that this difference is apparent after wearing the splints for only two weeks. They prove that 
the neutral angle wrist splint will provide better symptom relief than the traditional $20^{\circ}$ extension wrist splint or even in flexion. Even though, a customized orthosis should be the best option for the most effective treatment of the patient's symptoms.

Within the pharmacological treatment, using local corticosteroids, as methylprednisolone, was common. Oral corticosteroids reduce oedema, improving the space between the carpal tunnel and the median nerve [5]. It shows to have strong evidence to improve reported patients outcome [53]. Solely a greater dose of corticosteroids was found to be statistically significant for function after three months [32]. However, it was not more effective than shock waves after 24 weeks [24], or than a 5\% dextrose solution after 6 months, at the level of symptoms and function [36]. Its effect was more beneficial combined with a splint therapy than solely with a splint after 12 weeks, at the level of function, symptoms and electrophysiological parameters [54], with a fairly good quality of studies. Injection of dextrose is a new treatment for peripheral neuropathies, because it possesses osmolarity similar to that of normal saline, and no harmful effects have been reported from animal and human studies [55]. Furthermore, one dose of 5\% dextrose showed to be effective to improve function and CTS symptoms. Similar to a glucose molecule, the mechanism of an injection of hypo-osmolar 5\% dextrose postulated induced analgesic effect on tender peripheral nerves as well as central nerve system by osmotic rupture of local cells $[56,57]$. Another treatment growing in popularity is injecting platelet-rich plasma. This is used as an alternative to surgery because of the regenerative effect it can produce in patients with demyelination and axonal degeneration in CTS [48]. Platelet-rich plasma seems to be more effective three months after the injection at the level of symptoms, function and electrophysiological and clinical parameters than methylprednisolone injection [34]. However, there are no evidences indicating that it is more effective than night splinting after 10 weeks of treatment [33].

Considering electrotherapy, few studies assessed its effectiveness during its isolated application rather than combined with other type of therapies (manual therapy, splint, bandaging). This makes it difficult to evaluate the effectiveness of electrotherapy alone. Electro-acupuncture was the only treatment compared alone with night splinting; it obtained superior improvements in pain following five weeks of treatment compared with splinting [41]. However, the quality of the study was moderate. Low-power laser combined with bandaging [39], the use of diathermy and nerve gliding exercises [40] and ultrasound with ketoprofen gel and night splinting [48] presented better improvements in symptoms and function following treatment than these procedures alone or compared with placebo.

Focusing on manual therapy, all the techniques were assessed in combination or with other types of treatment (splints, bandages, diathermy, exercises). It seems that carpal bone mobilizations combined with night splinting are more effective after treatment at the level of function and symptoms than splinting alone. However, the methodological quality of these studies was moderate. It also seems that neurodynamic techniques used alone improve electrophysiological variables of function and symptoms compared with a control group, with moderate methodological quality [28]. Furthermore, neurodynamic techniques combined with other therapy, soft tissue or joint mobilization was more effective in symptoms and function following treatment than the combination of transcutaneous electrical nerve stimulation (TENS) and ultrasound or laser and ultrasound [30]; following treatment, acupuncture combined with splinting seems to be more effective for symptoms than splinting alone or combined with ibuprofen $[28,44]$. Acupuncture plus splinting also improved electrophysiological variables and function, in a study having good methodological quality [44]. Following treatment, paraffin combined with splinting and exercises seems to have a positive effect on function and electrophysiological parameters, but the study had moderate methodological quality [50]. All these treatments seem to have short term treatment effects, but few studies assessed their mid or long-term effect. Consequently, their effect on resolving CTS cannot be established.

The variability while performing the different manual techniques between studies can explain the diversity of results. Firstly, there is a lack of terminology consensus or 
standardization in the techniques whose aim is to mobilize the nervous system. Moreover, parameters such as the mobilization dosages, the number of joints to be mobilized and the consideration to stabilize or not the wrist joint while performing the gliding mobilization techniques are not uniform throughout the studies. Shacklock (2005) considers the stabilization of the related joint to the nerve region to be mobilized important to achieve a specific mobilization. On the other hand, joint mobilization aims to improve wrist range of motion. However, some joint mobilizations [58], described as transverse and ventral glide on dorsal side of the first carpal row, have been designed to release carpal tunnel syndrome, increasing cross-sectional area of carpal tunnel. In addition, soft tissue mobilization aims to reduce pressure on the carpal tunnel syndrome by improving the mobility of the myofascial tissues adjacent to the nerve. More studies need to be carried out to see the most effective combination of soft tissue and joint mobilization treatment in CTS patients.

Lavender oil is frequently used as an herbal medication for a wide variety of diseases, either topically or by inhalation. In vivo studies have shown that the active ingredients of lavender oil have important anti-inflammatory, anti-nociceptive, sedative, and analgesic therapeutic potentials [59]. However, using lavender ointments combined with night splints was not any more effective than a placebo ointment combined with splinting [49]. Results were only reported pre- and post-intervention, without follow-ups.

When severe patients were included, the isolated effect that conservative treatments produce in these patients was not found. These patients with electrophysiological abnormalities could show thenar muscle atrophy, and more diffuse symptoms. Surgical approach was the option of choice [5]. In some studies, electrotherapy was applied in mild, moderate and severe cases. Combined with a night splint, it seemed effective for symptoms and function following treatment; however, it is unclear whether these effects will be maintained at long-term [22,25]. In patients with severe CTS, other studies reported the effect of manual therapy including nerve gliding exercises [26], tendon gliding exercises [27] and acupuncture [28]. Both tendon and nerve gliding exercises improved function immediately and six months after treatment but combined with paraffin [27] and splinting or an exercise program [26]. Acupuncture reduced pain following treatment, once again combined with night splinting [28]. The moderate quality of these studies and the combination with other types of treatment make it difficult to establish the best effect of these therapies on the more severe CTS cases. It would be interesting to report results stratifying level of CTS severity to prove that conservative management it is a beneficial option for these patients. Still, electrotherapy and manual therapy could be beneficial to severe CTS.

Diagnosis of CTS should be performed by physical examination and nerve conduction studies [53]. All the studies included in this review performed one or both items. Electrophysiological studies were considered the gold standard to diagnose CTS patients. These tests can define the degree of demyelination and axonal loss that has occurred. Bland [60] scale described degrees to classify the severity of CTS as: Grade 0 denotes no neurophysiological abnormality; Grade 1, very mild CTS, detected only in two sensitive test; Grade 2, mild CTS as orthodromic sensory conduction velocity from index finger $<40 \mathrm{~m} / \mathrm{s}$ with motor terminal latency from wrist to abductor pollicis brevis (APB); Grade 3 , moderately severe CTS as motor terminal latency $>4.5 \mathrm{~ms}$ and $<6.5 \mathrm{~ms}$ with preserved index finger sensory nerve action potential (SNAP); Grade 4, severe CTS as motor terminal latency $>4.5 \mathrm{~ms}$ and $<6.5 \mathrm{~ms}$ with absent SNAP; Grade 5, very severe CTS as motor terminal latency $>6.5 \mathrm{~ms}$; and Grade 6, extremely severe CTS as surface motor potential from APB $<0.2 \mathrm{mV}$ peak-to-peak. However, the American Association of Orthopedic Surgeon [61] bundle this classification in mild (grade 1 and 2), moderate (grade 3) and severe (grade 4,5 , and 6), as reviewed studies performed. In the other hand, physical examination was performed in all studies reviewed, nonetheless a variety of test was performed. Phalen test, Tinel test and symptoms in neuroanatomical median nerve distribution were the most performed. Similarly, to electrophysiological studies, the physical examination tends to test the myelinated fiber function. Nowadays, this criterion should be disputed due to negative electrophysiological parameters in presence of CTS or symptoms beyond neuroanatomical 
distribution [7]. A new approach proposed to include the assessment of small nerve fibers or unmyelinated nerve fibers such as quantitative sensory testing to help confirming the presence of CTS [62]. This includes assessment of heat, cold and vibration sensation thresholds to evaluate nerve fibers that are not tested during nerve electrophysiological studies. Besides, combination of all these items must be taken into consideration in order to make a correct diagnosis, relating it to the patient's clinical history.

Another point is that, although CTS is generally idiopathic, some conditions such as diabetes or hypertension predispose individuals to suffer from neuropathy. In these patients, the risk of suffering CTS is three times greater than in healthy patients [63]. However, in studies including patients with hypertension and/or diabetes (only 3 of 29), they only represented from $5 \%$ to $20 \%$ of all the patients evaluated [21,22]. Consequently, the direct effect of electrotherapy in patients with hypertensions and/or diabetes cannot be established. There was only a single study in the review [23] that studied patients with diabetes and CTS. It was found that, after four weeks of treatment, manual therapy (soft tissue and nerve mobilization) seemed more effective than the use of TENS and ultrasound on function, symptoms and neurodynamic results. However, this study did not present long-term results and its methodological quality was only moderate. Assessing small fibers nerve could help therapist to perform a more comprehensive approach on patients with both CTS and systemic condition. Even though, a combined protocol of manual techniques could be more effective for diabetic patients with CTS than electrotherapy in the short term.

We believe that there is insufficient research on CTS in patients with systemic pathology. There is a lack of representation of CTS patients with diabetes in the studies given their high prevalence and the possibility of developing CTS throughout their lives. Moreover, the conservative approach should be more studied in this subgroup as it can be more difficult to diagnose CTS in patients with diabetes because the earliest symptoms can be mistaken for diabetic polyneuropathy [64], and controversy exists about surgical treatment for these patients [65]. Given the lack of representation of patients with CTS and systemic diseases in the studies dealing with conservative therapy approaches, we consider this type of patients should be included in the sample selection criteria in studies on conservative treatment for CTS. This would help to ascertain the effect of conservative treatment in more representative CTS samples.

There are some limitations in this review that arise from the biases found in the articles. The lack of uniformity in the sample selection criteria makes it difficult to compare data among them. The variability of techniques proposed, and their combinations do not permit accurate comparison of their effectiveness either.

The lack of uniformity in methodological quality also hinders accurately evaluating treatment effectiveness. In addition, there might be references in other language besides Spanish, English and French that were not considered due to the language restrictions established in our selection criteria. More reviews should be performed performing quantitative analysis to prove specific results.

\section{Conclusions}

In patients with mild and moderate CTS, electrotherapy seems to be effective following treatment as long as it is combined with splinting, but its effectiveness is no greater than manual therapy. Corticosteroid injection does not appear to be more effective than saline solution or other techniques such as electrotherapy or manual therapy. No conclusions can be reached on the effectiveness of platelet-rich plasma to treat CTS; further studies are needed. One injection of 5\% dextrose seems to be effective for CTS symptoms and function. Wrist splint could be useful to complement treatments, however wrist position should be described in future studies, taking into account the position that best relieves patient's symptoms. Electrotherapy and manual therapy could be beneficial for severe CTS, and manual therapy for diabetic CTS in the short term. However, the specific effect of conservative treatment should be established with a stratification analysis depending on the intensity level of CTS. More long-term studies need to be carried out to specify which 
treatment is most beneficial for which type of severity. It seems that some pharmacological treatments, manual therapy and electrotherapy have benefits for handling CTS, although the most effective combination of techniques is unknown. However, it does seem that a combined treatment for each patient might be the most effective option.

A final point is that patients with systemic conditions and severe CTS should be included in the inclusion criteria for future CTS studies analyzing conservative approaches.

Author Contributions: Search process, M.H.-S., R.M.-C. and C.H.-G.; methodology, M.H.-S., R.M.-C. and C.H.-G.; qualitative analysis, M.H.-S., R.M.-C. and C.H.-G.; resources, C.H.-G. and M.O.L.-L.; data curation, C.H.-G. and M.O.L.-L.; writing—original draft preparation, M.H.-S.; writing-review and editing, C.H.-G., M.O.L.-L. and J.R.-S.; visualization, C.H.-G., M.O.L.-L., J.C.-d.-T., S.M.-B., S.H.-A. and J.R.-S.; supervision, C.H.-G., M.O.L.-L., J.C.-d.-T., S.M.-B., S.H.-A. and J.R.-S.; project administration, C.H.-G., M.O.L.-L. and J.M.T.-M. All authors have read and agreed to the published version of the manuscript.

Funding: This research received no external funding.

Institutional Review Board Statement: Not applicable.

Informed Consent Statement: Not applicable.

Conflicts of Interest: The authors declare no conflict of interest. The funders had no role in the design of the study; in the collection, analyses, or interpretation of data; in the writing of the manuscript, or in the decision to publish the results.

\section{References}

1. Wipperman, J.; Goerl, K. Diagnosis and management of carpal tunnel syndrome. J. Musculoskelet. Med. 2016, $94,47-60$.

2. Atroshi, I.; Gummesson, C.; Johnsson, R.; Ornstein, E.; Ranstam, J.; Rosén, I. Prevalence of Carpal Tunnel Syndrome in a General Population. JAMA 1999, 282, 153-158. [CrossRef] [PubMed]

3. Zhuang, T.; Kortlever, J.T.; Shapiro, L.M.; Baker, L.; Harris, A.H.; Kamal, R.N. The Influence of Cost Information on Treatment Choice: A Mixed-Methods Study. J. Hand Surg. 2020, 45, 899-908.e4. [CrossRef] [PubMed]

4. $\quad$ Erickson, M.; Lawrence, M.; Stegink Jansen, C.; Coker, D.; Amadio, P.; Cleary, C. Carpal Tunnel Syndrome: A Summary of Clinical Practice Guideline Recommendations-Using the Evidence to Guide Physical Therapist Practice. J. Orthop. Sports Phys. Ther. 2019, 49, 359-360.

5. Padua, L.; Coraci, D.; Erra, C.; Pazzaglia, C.; Paolasso, I.; Loreti, C.; Caliandro, P.; Hobson-Webb, L.D. Carpal tunnel syndrome: Clinical features, diagnosis, and management. Lancet Neurol. 2016, 15, 1273-1284. [CrossRef]

6. $\quad$ Lin, Y.-N.; Chiu, C.-C.; Huang, S.-W.; Hsu, W.-Y.; Liou, T.-H.; Chen, Y.-W.; Chang, K.-H. Association between Manual Loading and Newly Developed Carpal Tunnel Syndrome in Subjects with Physical Disabilities: A Follow-Up Study. Arch. Phys. Med. Rehabilit. 2017, 98, 2002-2008. [CrossRef] [PubMed]

7. Schmid, A.B.; Fundaun, J.; Tampin, B. Entrapment neuropathies: A contemporary approach to pathophysiology, clinical assessment, and management. PAIN Rep. 2020, 5, e829. [CrossRef]

8. Moradi, A.; Sadr, A.; Ebrahimzadeh, M.H.; Hassankhani, G.G.; Mehrad-Majd, H. Does diabetes mellitus change the carpal tunnel release outcomes? Evidence from a systematic review and meta-analysis. J. Hand Ther. 2020, 1-7. [CrossRef]

9. Pourmemari, M.H.; Shiri, R. Diabetes as a risk factor for carpal tunnel syndrome: A systematic review and meta-analysis. Diabet. Med. 2016, 33, 10-16. [CrossRef] [PubMed]

10. Barbosa, J.; Castro, G.; Rojas, W.; Palacios, E. Frecuencia y evolución de la polineuropatía en pacientes hipotiroideos no controlados. Acta Neurol. Colomb. 2002, 18, 83-87.

11. Ibrahim, I.; Khan, W.; Goddard, N.; Smitham, P. Carpal Tunnel Syndrome: A Review of the Recent Literature. Open Orthop. J. 2012, 6, 69-76. [CrossRef]

12. Zyluk-Gadowska, P.; Zyluk, A. Factors Affecting the Outcomes of Carpal Tunnel Surgery: A Review. Handchir. Mikrochir. Plast. Chir. 2016, 48, 260-265. [CrossRef] [PubMed]

13. Jiménez del Barrio, S.; Bueno Gracia, E.; Hidalgo García, C.; Estébanez de Miguel, E.; Tricás Moreno, J.M.; Rodríguez Marco, S. Tratamiento conservador en pacientes con síndrome del túnel carpiano con intensidad leve o moderada. Revisión sistemática. Neurología 2018, 33, 590-601. [CrossRef] [PubMed]

14. Zyluk, A.; Strychar, J. Results of the treatment of carpal tunnel syndrome-A review. Chir. Narzadow. Ruch. Ortop. Polska 2005, 70, 439-445.

15. Perkins, B.A.; Olaleye, D.; Bril, V. Carpal Tunnel Syndrome in Patients with Diabetic Polyneuropathy. Diabetes Care 2002, 25, 565-569. [CrossRef] [PubMed]

16. Afshar, A.; Tabrizi, A.; Tajbakhsh, M.; Navaeifar, N. Subjective Outcomes of Carpal Tunnel Release in Patients with Diabetes and Patients without Diabetes. J. Hand Microsurg. 2020, 12, 183-188. [CrossRef] 
17. Liberati, A.; Altman, D.; Tetzlaff, J.; Mulrow, C.; Gotzsche, P.; Ioannidis, J. The PRISMA statement for reporting systematic reviews and meta-analyses of studies that evaluate health care interventions: Explanation and elaboration. PLoS Med. 2009, 6, 1-2. [CrossRef]

18. Leite, J.C.D.C.; Jerosch-Herold, C.; Song, F. A systematic review of the psychometric properties of the Boston Carpal Tunnel Questionnaire. BMC Musculoskelet. Disord. 2006, 7, 78-79. [CrossRef]

19. Herbert, R.; Moseley, A.; Sherrington, C.; Maher, C. Escala PEDro-Español. Physiotherapy 2000, 86, 55. [CrossRef]

20. Sterne, J.A.C.; Savović, J.; Page, M.J.; Elbers, R.G.; Blencowe, N.S.; Boutron, I.; Cates, C.J.; Cheng, H.-Y.; Corbett, M.S.; Eldridge, S.M.; et al. RoB 2: A revised tool for assessing risk of bias in randomised trials. BMJ 2019, 366, 14898. [CrossRef]

21. Wu, Y.-T.; Ke, M.-J.; Chou, Y.-C.; Chang, C.-Y.; Lin, C.-Y.; Li, T.-Y.; Shih, F.-M.; Chen, L.-C. Effect of radial shock wave therapy for carpal tunnel syndrome: A prospective randomized, double-blind, placebo-controlled trial. J. Orthop. Res. 2015, 34, 977-984. [CrossRef]

22. Chen, L.C.; Ho, C.W.; Sun, C.H.; Lee, J.T.; Li, T.Y.; Shih, F.M. Ultrasound-Guided Pulsed Radiofrequency for Carpal Tunnel Syndrome: A Single-Blinded Randomized Controlled Study. PLoS ONE 2015, 10, e0129918. [CrossRef] [PubMed]

23. Talebi, G.A.; Saadat, P.; Javadian, Y.; Taghipour, M. Manual therapy in the treatment of carpal tunnel syndrome in diabetic patients: A randomized clinical trial. Casp. J. Intern. Med. 2018, 9, 283-289.

24. Atthakomol, P.; Manosroi, W.; Phanphaisarn, A.; Phrompaet, S.; Iammatavee, S.; Tongprasert, S. Comparison of single-dose radial extracorporeal shock wave and local corticosteroid injection for treatment of carpal tunnel syndrome including mid-term efficacy: A prospective randomized controlled trial. BMC Musculoskelet. Disord. 2018, 19, 32. [CrossRef] [PubMed]

25. Chung, V.C.H.; Ho, R.S.T.; Liu, S.; Chong, M.K.C.; Leung, A.W.N.; Yip, B.H.K. Electroacupuncture and splinting versus splinting alone to treat carpal tunnel syndrome: A randomized controlled trial. Can. Med. Assoc. J. 2016, 188, 867-875. [CrossRef]

26. Hamzeh, H.; Madi, M.; Alghwiri, A.A.; Hawamdeh, Z. The long term effect of neurodynamics vs. exercise therapy on pain and function in people with carpal tunnel syndrome: A randomized parallel-group clinical trial. J. Hand Ther. 2020, 20, 30144-30147. [CrossRef]

27. Horng, Y.S.; Hsieh, S.F.; Tu, Y.K.; Lin, M.C. The comparative effectiveness of tendon and nerve gliding exercises in patients with carpal tunnel syndrome: A randomized trial. Am. J. Phys. Med. Rehabil. 2011, 90, 435-442. [CrossRef] [PubMed]

28. Tezel, N.; Umay, E.; Yllmaz, V.; Cakci, A. Acupuncture plus night splint for quality of life and disability in patients with carpal tunnel syndrome: A randomized controlled trial. Integr. Med. Res. 2019, 8, 284-288. [CrossRef] [PubMed]

29. Wu, Y.-T.T.; Ho, T.-Y.Y.; Chou, Y.-C.C.; Ke, M.-J.J.; Li, T.-Y.Y.; Tsai, C.-K.K. Six-month Efficacy of Perineural Dextrose for Carpal Tunnel Syndrome: A Prospective, Randomized, Double-Blind, Controlled Trial. Mayo Clin. Proc. 2017, 92, 1179-1189. [CrossRef]

30. Wolny, T.; Saulicz, E.; Linek, P.; Shacklock, M.; Myśliwiec, A.; Wolny, T. Efficacy of Manual Therapy Including Neurodynamic Techniques for the Treatment of Carpal Tunnel Syndrome: A Randomized Controlled Trial. J. Manip. Physiol. Ther. 2017, 40, 263-272. [CrossRef] [PubMed]

31. Wu, Y.-T.; Ho, T.-Y.; Chou, Y.-C.; Ke, M.-J.; Li, T.-Y.; Huang, G.-S.; Chen, L.-C. Six-month efficacy of platelet-rich plasma for carpal tunnel syndrome: A prospective randomized, single-blind controlled trial. Sci. Rep. 2017, 7, 1-11. [CrossRef] [PubMed]

32. Karimzadeh, A.; Bagheri, S.; Raeissadat, S.A.; Bagheri, S.; Rayegani, S.M.; Rahimi-Dehgolan, S.; Safdari, F.; Abrishamkarzadeh, H.; Shirzad, H. The comparison of the effectiveness between different doses of local methylprednisolone injection versus triamcinolone in Carpal Tunnel Syndrome: A double-blind clinical trial. J. Pain Res. 2019, 12, 579-584. [CrossRef] [PubMed]

33. Raeissadat, S.A.; Karimzadeh, A.; Hashemi, M.; Bagherzadeh, L. Safety and efficacy of platelet-rich plasma in treatment of carpal tunnel syndrome; a randomized controlled trial. BMC Musculoskelet. Disord. 2018, 19, 49. [CrossRef] [PubMed]

34. Senna, M.K.; Shaat, R.M.; Ali, A.A.A. Platelet-rich plasma in treatment of patients with idiopathic carpal tunnel syndrome. Clin. Rheumatol. 2019, 38, 3643-3654. [CrossRef] [PubMed]

35. Wang, J.C.; Liao, K.K.; Lin, K.-P.; Chou, C.-L.; Yang, T.-F.; Huang, Y.-F. Efficacy of Combined Ultrasound-Guided Steroid Injection and Splinting in Patients with Carpal Tunnel Syndrome: A Randomized Controlled Trial. Arch. Phys. Med. Rehabil. 2017, 98, 947-956. [CrossRef]

36. Wu, Y.-T.; Ke, M.-J.; Ho, T.-Y.; Li, T.-Y.; Shen, Y.-P.; Chen, L.-C. Randomized double-blinded clinical trial of 5\% dextrose versus triamcinolone injection for carpal tunnel syndrome patients. Ann. Neurol. 2018, 84, 601-610. [CrossRef] [PubMed]

37. Çatalbas, N.; Akkaya, N.; Atalay, N.S.; Sahin, F. Ultrasonographic imaging of the effects of continuous, pulsed or sham ultrasound treatments on carpal tunnel syndrome: A randomized controlled study. J. Back Musculoskelet. Rehabil. 2018, 31, 981-989. [CrossRef] [PubMed]

38. Fusakul, Y.; Aranyavalai, T.; Saensri, P.; Thiengwittayaporn, S. Low-level laser therapy with a wrist splint to treat carpal tunnel syndrome: A double-blinded randomized controlled trial. Lasers Med. Sci. 2014, 29, 1279-1287. [CrossRef]

39. Güner, A.; Altan, L.; Aksoy, M.K. The effectiveness of the low-power laser and kinesiotaping in the treatment of carpal tunnel syndrome, a pilot study. Rheumatol. Int. 2018, 38, 895-904. [CrossRef]

40. Incebiyik, S.; Boyaci, A.; Tutoglu, A. Short-term effectiveness of short-wave diathermy treatment on pain, clinical symptoms, and hand function in patients with mild or moderate idiopathic carpal tunnel syndrome. J. Back Musculoskelet. Rehabil. 2015, 28, 221-228. [CrossRef]

41. Kumnerddee, W.; Kaewtong, A. Efficacy of acupuncture versus night splinting for carpal tunnel syndrome: A randomized clinical trial. J. Med. Assoc. Thail. 2010, 93, 1463-1469. 
42. Geler Külcü, D.; Bursali, C.; Aktaş, İ.; Bozkurt Alp, S.; Ünlü Özkan, F.; Akpinar, P. Kinesiotaping as an alternative treatment method for carpal tunnel syndrome. Turk. J. Med. Sci. 2016, 46, 1042-1049. [CrossRef] [PubMed]

43. Gunay, B.; Alp, A. The effectiveness of carpal bone mobilization accompanied by night splinting in idiopathic carpal tunnel syndrome. Turkish. J. Phys. Med. Rehabil. 2015, 61, 45-50. [CrossRef]

44. Hadianfard, M.; Bazrafshan, E.; Momeninejad, H.; Jahani, N. Efficacies of Acupuncture and Anti-inflammatory Treatment for Carpal Tunnel Syndrome. J. Acupunct. Meridian Stud. 2015, 8, 229-235. [CrossRef] [PubMed]

45. Wolny, T.; Linek, P. Is manual therapy based on neurodynamic techniques effective in the treatment of carpal tunnel syndrome? A randomized controlled trial. Clin. Rehabil. 2018, 33, 408-417. [CrossRef]

46. Dinarvand, V.; Abdollahi, I.; Raeissadat, S.A.; Bandpei, M.A.M.; Babaee, M.; Talimkhani, A. The Effect of Scaphoid and Hamate Mobilization on Treatment of Patients with Carpal Tunnel Syndrome. Anesthesiol. Pain Med. 2017, 7, e14621. [CrossRef]

47. Xu, D.; Ma, W.; Jiang, W.; Hu, X.; Jiang, F.; Mao, C.; Wang, Y.; Fang, L.; Luo, N.; Li, H.; et al. A randomized controlled trial: Comparing extracorporeal shock wave therapy versus local corticosteroid injection for the treatment of carpal tunnel syndrome. Int. Orthop. 2020, 44, 141-146. [CrossRef] [PubMed]

48. Yildiz, N.; Atalay, N.S.; Gungen, G.O.; Sanal, E.; Akkaya, N.; Topuz, O. Comparison of ultrasound and ketoprofen phonophoresis in the treatment of carpal tunnel syndrome. J. Back Musculoskelet. Rehabil. 2011, 24, 39-47. [CrossRef]

49. Eftekharsadat, B.; Roomizadeh, P.; Torabi, S.; Heshmati-Afshar, F.; Jahanjoo, F.; Babaei-Ghazani, A. Effectiveness of Lavendula stoechas essential oil in treatment of mild to moderate carpal tunnel syndrome: A randomized controlled trial. J. Hand Ther. 2018, 31, 437-442. [CrossRef]

50. Ordahan, B.; Karahan, A.Y. Efficacy of paraffin wax bath for carpal tunnel syndrome: A randomized comparative study. Int. J. Biometeorol. 2017, 61, 2175-2181. [CrossRef]

51. Hall, B.; Lee, H.C.; Fitzgerald, H.; Byrne, B.; Barton, A.; Lee, A.H. Investigating the effectiveness of full-time wrist splinting and education in the treatment of carpal tunnel syndrome: A randomized controlled trial. Am. J. Occup. Ther. 2013, 67, 448-459. [CrossRef] [PubMed]

52. Burke, D.T.; Burke, M.M.; Stewart, G.W.; Cambré, A. Splinting for carpal tunnel syndrome: In search of the optimal angle. Arch. Phys. Med. Rehabil. 1994, 75, 1241-1244. [CrossRef]

53. American Academy of Orthopaedic Surgeons Boards of Directors. Management of carpal tunnel syndrome. Am. Acad. Orthop. Surg. 2016, 35, 1-983.

54. Huisstede, B.M.; Hoogvliet, P.; Randsdorp, M.S.; Glerum, S.; van Middelkoop, M.; Koes, B.W. Carpal Tunnel Syndrome. Part I: Effectiveness of Nonsurgical Treatments-A Systematic Review. Arch. Phys. Med. Rehabil. 2010, 91, 981-1004. [CrossRef]

55. Huisstede, B.M.; Fridén, J.; Coert, J.H.; Hoogvliet, P. Carpal tunnel syndrome: Hand surgeons, hand therapists, and physical medicine and rehabilitation physicians agree on a multidisciplinary treatment guidelined-results from the European HANDGUIDE study. Arch. Phys. Med. Rehabil. 2014, 95, 2253-2263. [CrossRef]

56. Maniquis-Smigel, L.; Reeves, K.D.; Rosen, H.J.; Lyftogt, J.; Graham-Coleman, C.; Cheng, A.-L.; Rabago, D. Short Term Analgesic Effects of 5\% Dextrose Epidural Injections for Chronic Low Back Pain: A Randomized Controlled Trial. Anesthesiol. Pain Med. 2016, 7, e42550. [CrossRef]

57. Rabago, D.; Slattengren, A.; Zgierska, A. Prolotherapy in Primary Care Practice. Prim. Care Clin. Off. Pract. 2010, 37, 65-80. [CrossRef]

58. Bueno-Gracia, E.; Ruiz-De-Escudero-Zapico, A.; Malo-Urriés, M.; Shacklock, M.; Estébanez-De-Miguel, E.; Fanlo-Mazas, P.; Caudevilla-Polo, S.; Jiménez-Del-Barrio, S. Dimensional changes of the carpal tunnel and the median nerve during manual mobilization of the carpal bones. Musculoskelet. Sci. Pract. 2018, 36, 12-16. [CrossRef]

59. da Silva, G.L.; Luft, C.; Lunardelli, A.; Amaral, R.H.; da Silva Melo, D.A.; Donadio, M.V.F. Antioxidant, analgesic and antiinflammatory effects of lavender essential oil. An. Acad. Bras. Cienc. 2015, 87, 1397-1408. [CrossRef]

60. Bland, J.D.P. A neurophysiological grading scale for carpal tunnel syndrome. Muscle Nerve 2000, 23, 1280-1283. [CrossRef]

61. Jablecki, C.K.; Andary, M.T.; Floeter, M.K.; Miller, R.G.; Quartly, C.A.; Vennix, M.J. Practice parameter for electrodiagnostic studies in carpal tunnel syndrome: Summary statement. Muscle Nerve 2002, 25, 918-922.

62. Schmid, A.B.; Bland, J.D.P.; Bhat, M.A.; Bennett, D.L.H. The relationship of nerve fibre pathology to sensory function in entrapment neuropathy. Brain 2014, 137, 3186-3199. [CrossRef] [PubMed]

63. Karpitskaya, Y.; Novak, C.B.; MacKinnon, S.E. Prevalence of Smoking, Obesity, Diabetes Mellitus, and Thyroid Disease in Patients with Carpal Tunnel Syndrome. Ann. Plast. Surg. 2002, 48, 269-273. [CrossRef] [PubMed]

64. Zhang, D.; Collins, J.; Blazar, P.; Earp, B.E. Factors Associated with Advanced Presentation for Carpal Tunnel Release. J. Hand Surg. 2020, 45, 111-116. [CrossRef] [PubMed]

65. Westenberg, R.F.; Oflazoglu, K.; De Planque, C.A.; Jupiter, J.B.; Eberlin, K.R.; Chen, N.C. Revision Carpal Tunnel Release: Risk Factors and Rate of Secondary Surgery. Plast. Reconstr. Surg. 2020, 145, 1204-1214. [CrossRef] 